Research Article

\title{
Exzellenz in der österreichischen und deutschen Molkereibranche - ein Vergleich
}

\section{Excellence in the Austrian and German Dairy Sector - a comparison}

\author{
Beate Gebhardt ${ }^{1}$, Ingrid Deixler ${ }^{2}$, Imke Wißmann ${ }^{1}$, Julia Anna Jungmair ${ }^{2}$, Siegfried Pöchtrager ${ }^{2}$
}

\author{
${ }^{1}$ Universität Hohenheim, Fachgebiet für Agrarmärkte (420B), Schwerzstraße 46, 70599 Stuttgart, Deutschland \\ ${ }^{2}$ Universität für Bodenkultur Wien, Institut für Marketing und Innovation, Feistmantelstraße 4, 1180 Wien, Österreich \\ Korrespondierende Autorin: beate.gebhardt@uni-hohenheim.de
}

Einreichung: 26. Juli 2019, überarbeitete Einreichung: 25. Mai 2020, Annahme: 29. Juni 2020

\section{Zusammenfassung}

Um als herausragendes Unternehmen wahrgenommen zu werden, müssen Unternehmen über ihre Aktivitäten oder Produkte kommunizieren. Die Darstellung von Exzellenz, im Sinne von Spitzenleistung und Bessersein als Andere, bietet Unternehmen die Möglichkeit sich abzuheben. Auf Basis von Länderstudien in Österreich und Deutschland wird im vorliegenden Beitrag Exzellenz sowie die Kommunikation darüber in der Molkereibranche verglichen. Die Thematisierung von Nachhaltigkeit (Selbstberichte) sowie das Aufzeigen von Prämierungen und Zertifizierungen (Drittberichte) werden dabei als Darstellungsoptionen für Exzellenz herangezogen. Die Auswertung von 22 in Österreich und Deutschland geführten Experteninterviews erfolgte mittels kategorialer Inhaltsanalyse nach Mayring. Die deskriptive Website-Analyse von 197 österreichischen und deutschen Molkereien ergänzt die Studie.

Die Ergebnisse zeigen, dass es steigende Produktanforderungen sowie Konkurrenz- und Preisdruck für Molkereien beider Länder unumgänglich machen, sich durch hervorragende Leistungen und klare Differenzierung am Markt abzuheben. Exzellenz in der Molkereibranche wird als multikausale und relative Kenngröße beschrieben, in die sich Nachhaltigkeit einordnet. Prämierungen und Zertifikate haben dabei unterschiedliches Gewicht. In Österreich ist die Kommunikation über gewonnene Prämierungen ausgeprägter als in Deutschland. In beiden Ländern werden vor allem Produktprämierungen gezeigt. Zertifikate gelten in beiden Ländern nur eingeschränkt als Zeichen von Exzellenz. Insgesamt stehen diese Drittberichte meist in Abhängigkeit von finanziellen und personellen Ressourcen; weniger gilt dies für Selbstberichte.

Schlagworte: Exzellenz, Inhaltsanalyse nach Mayring, Kommunikation, Nachhaltigkeit, Molkereibranche, Prämierung, Zertifizierung

\section{Summary}

Companies must communicate about their activities or products to be perceived as an outstanding company. Representing excellence, in terms of "top performance" and "outperforming others", offers companies the opportunity to differentiate themselves from their competitors. The present article compares the Austrian view on excellence and its communication in the dairy industry with the German one by means of two regional studies. To this end, their focus on sustainability (self-reports) and the presentation of awards and certifications (third-party reports) are used as expressions of excellence. We evaluated 22 expert interviews conducted in Austria and Germany using Mayring's qualitative content analysis at category level. The descriptive website analysis of 197 Austrian and German dairies complements the study.

The results show that dairies from both countries must stand out from the market by performing outstandingly and differentiating themselves due to increasing product requirements and competitive pressure. Excellence in the dairy industry is described as a multicausal and relative parameter in which sustainability is aligned. In this context, the communication of awards and certificates have different weights. Austrian dairies communicate won awards more clearly than German. In both countries mainly product awards are displayed; and certificates are considered signs of excellence only to a limited extent. Overall, external reports are mostly dependent on financial and human resources; to a lesser extent this applies to self-reports.

Keywords: award, certification, communication, dairy industry, excellence, Mayring content analysis, sustainability 


\section{Einleitung}

Der Markt für Lebensmittel gilt in Österreich (Ö) und Deutschland (D) als weitestgehend gesättigt und von einer stagnierenden oder nur wenig steigenden Nachfrage, einem hohen Konkurrenzdruck sowie einem aggressiven Preiskampf gekennzeichnet. Dies wird ebenfalls für die Milchwirtschaft berichtet: Einem vielfältigen und vielzähligen Angebot an Milch- und Molkereierzeugnissen stehen in Österreich eine insgesamt nur gering steigende Nachfrage (BMLFUW, 2016) und in Deutschland ein nahezu stagnierender Pro-Kopf-Konsum von Frischmilcherzeugnissen (MIV, 2017) gegenüber. Gleichzeitig wachsen Anforderungen an die Produktqualität und Warensicherheit seitens des Lebensmitteleinzelhandels (LEH) sowie der Konsumenten und Konsumentinnen (Schmid et al., 2011; BMLFUW, 2017; MIV, 2017). Insbesondere ökologische und soziale Aspekte im Sinne des Leitbilds einer nachhaltigen Entwicklung gewinnen an Bedeutung (Albersmeier et al., 2008; Böhm et al., 2009).

Strukturelle Herausforderungen sowie sinkende Umsatzrenditen und damit ein geringerer finanzieller Spielraum für Investitionen, Innovationen oder Werbung führen zur Notwendigkeit in der Unternehmensführung, Strategien zu entwickeln, um erfolgreich am Markt (weiter) bestehen zu können (Porter, 1985; Collins, 2001; Schmidpeter, 2016; Kuckertz et al., 2019). „Die Besten sein“ (Collins, 2001: 95) und eine solche herausragende Leistung oder Position gegenüber den Anspruchsgruppen deutlich sichtbar herauszustellen, können hierbei Ansätze sein, die unter dem Begriff „Exzellenz" beschrieben werden (DahlgaardPark und Dahlgaard, 2006; Brunner und Wagner, 2011; Huck-Sandhu, 2011; Wunder und Bausch, 2015). Exzellenz meint dabei Großartigkeit oder gar Brillanz und Spitzenleistung (Peters und Watermann, 1982). Dies umfasst eine exzellente Unternehmenstätigkeit (Wunder und Bausch, 2015) und gleichermaßen deren Darstellung (Mast, 2013). Gebhardt et al. (2019) unterscheiden demnach in „Exzellenzperformance “ und „Exzellenzkommunikation“. Exzellenz steht in engem Zusammenhang mit der Anwendung von Qualitätsmanagementsystemen und der Betrachtung von Erfolgsfaktoren. Dalluege et al. (2012) beschreiben, dass exzellente Organisationen dauerhaft herausragende Leistungen erzielen, die die Erwartungen aller ihrer Interessensgruppen erfüllen oder übertreffen. Um Nachfrage zu generieren, reichen auch aus Sicht der Marketingforschung „gute“ Produkte alleine oft nicht mehr aus, vielmehr müssen diese deutlich besser sein als die von der Konkurrenz angebotenen (Voeth und Herbst, 2013). Für Unternehmen der Molkereibranche gewinnt aus den genannten Gründen eine klare Positionierung als innovatives und erfolgreiches Unternehmen verstärkt an Bedeutung: Nach Weindlmaier (2004), Friedrich (2010) und Steffens (2013) betrifft diese Positionierung neben der Entscheidung über bestimmte Absatz- und Nischenmärkte auch die klare Abgrenzung von Konkurrenzprodukten in Form von Alleinstellungsmerkmalen (engl. unique selling point (USP)). Eine durch „emotionale Qualität“ gewonnene Differenzierung mittels produktbezogenem Zusatznutzen (engl. added value), wie gesellschaftliche Verantwortung oder Gesundheit (Schmid et al., 2011), zielt darauf $\mathrm{ab}$, die Anonymität der meist homogenen und demnach leicht austauschbaren Milch- und Molkereiprodukte zu überwinden (von Alvensleben, 2000; Winkelmann, 2004) und für die Konsumentinnen und Konsumenten authentische Produkte mit Herkunfts-, Hersteller- und/oder Produktionsbezug zu schaffen. Außerdem soll eine strategische Positionierung Vertrauen und Sympathie bei den Konsumenten und Konsumentinnen aufbauen und weiter steigern. In weiterer Folge kann der durch Produktattribute geschaffene Mehrwert die Kauf- und Zahlungsbereitschaft der Konsumenten und Konsumentinnen erhöhen (Steffens, 2013; VÖM, 2016). Qualität gilt als zentraler Erfolgsfaktor (Weindlmaier, 2004), welcher sich auf alle Ebenen des Unternehmens bezieht: Produkt, Prozess, Mitarbeiter, Lieferanten sowie die Beziehung zu den Kunden. Nachhaltigkeit und die Übernahme von gesellschaftlicher Verantwortung (engl. Corporate Social Responsibility (CSR)) gewinnen in diesem Zusammenhang stetig an Bedeutung (Veer und Teitscheid, 2010a, b; Flint et al., 2016); unter anderem auch, da Haltungsbedingungen und Umweltwirkungen in der Milchproduktion verstärkt in der öffentlichen Diskussion stehen (Wienert, 2007; Luhmann et al., 2017). Letztlich sind für die Mehrheit der Konsumenten Geschmack, teils auch Frische, Aussehen und Preis, weiterhin wesentliche Kriterien im Kaufentscheid von Lebensmitteln (MRI, 2008; Gebhardt, 2012; Moog und Gebhardt, 2018).

Was Unternehmen unter Exzellenz verstehen, wie sie dabei Nachhaltigkeit einordnen und Exzellenz kommunizieren, wird in diesem Beitrag für die Molkereibranche in Österreich und in Deutschland betrachtet. Eine entsprechende Untersuchung in der Ernährungswirtschaft und der Molkereibranche fehlt bisher. 


\section{Zielsetzung}

Die Vergleichsstudie hat zum Ziel, die Bedeutung von Exzellenzperformance und Exzellenzkommunikation aus Sicht der österreichischen (Ö) und der deutschen (D) Molkereibranche zu erfassen und gegenüberzustellen. Im weiteren Beitrag werden dazu folgende Forschungsfragen betrachtet:

1. Welche Faktoren umfasst Exzellenz aus Sicht von Unternehmen der Molkereibranche und inwieweit ordnet sich hierbei Nachhaltigkeit ein?

2. Welche unterschiedlichen Stellenwerte nehmen Selbstberichte und Drittberichte, wie Prämierungen und Zertifizierungen, in der Kommunikation über Exzellenz von Molkereien ein?

3. Worin unterscheiden sich die Sichtweisen und Darstellung von Exzellenz in der österreichischen und der deutschen Molkereibranche?

Expertenbefragungen sowie eine Website-Analyse von Molkereien in beiden Ländern wurden zur Beantwortung dieser Fragen durchgeführt. Für einen Ländervergleich prädestinieren die geografische Nähe und gemeinsame Sprache der beiden Nachbarländer Österreich und Deutschland. Dies verbindet deren Märkte und erhöht die Konkurrenz „vor der Haustür“. Mit Produktinnovationen und Qualitätsstandards, wie zertifiziert gentechnikfreie Milch (ARGE Gentechnik-frei, 2015) und Heumilch (VÖM, 2016), entwickelt sich die österreichische Molkereibranche seit einigen Jahren als Vorreiter, die Aufmerksamkeit und entsprechende Anpassungsstrategien in Deutschland nach sich ziehen. Die Ergebnisse können im Weiteren für Molkereivertretungen beider Länder handlungsleitend in der inländischen und internationalen Positionierung sein.

\section{Situation der Molkereibranche in Österreich und Deutschland}

Die Analyse der Molkereibranche in Österreich und Deutschland zeigt, dass sich die beiden Länder hinsichtlich ihrer bevorstehenden Aufgaben und den zu erwartenden Entwicklungen in dieser Branche durchaus ähneln:

Mit der zunehmenden Konzentration der Molkereien sowie gesteigerten Milchmengen, bei zeitgleichem Rückgang der land- und forstwirtschaftlichen Betriebe (Österreich: minus 3 Prozent von 166.317 (2013) auf 161.150 (2016); Deutschland: minus 1,5 Prozent von 285.200 (2013) auf
280.800 (2015), befindet sich die Molkereibranche beider Länder in einem wettbewerbsintensiven und globalisierten Absatzmarkt. Die Inlandsmärkte sind gesättigt, Auslandsmärkte sind wichtige Absatzkanäle für Milch und Milcherzeugnisse. Die Hälfte der deutschen Milchproduktion im Wert von 8,43 Mrd. Euro im Jahr 2016 ist für den Export bestimmt. Österreich exportierte 2016 Milch- und Milchprodukte im Wert von 1,17 Mrd. Euro (Schlickau et al., 2008; LEL, 2016; BMLFUW, 2017; MIV, 2017; Statistisches Bundesamt, 2017). Der Branchendruck wächst und wird durch die zunehmende Globalisierung für Milch und Milchprodukte (z. B. Ende der Milchquote 2015 und Wegfall der nationalen Marktordnung durch den EU-Beitritt Österreich im Jahr 1995) sowie weiteren strukturellen Entwicklungen (z. B. zunehmende Schnelllebigkeit von Innovationen, steigende Qualitäts- und Produktionsstandards, steigende Verhandlungsmacht des LEH und wachsende Konsumentenansprüche) verschärft (Schmid et al., 2011; Tribl und Salhofer, 2013; MIV, 2017).

Insbesondere in ihren Größenverhältnissen zeigen die Molkereisektoren beider Nachbarländer deutliche Unterschiede:

- Die Milchproduktion ist in Österreich kleinstrukturierter als in Deutschland und erfolgt zum Großteil in Berg- oder benachteiligten Gebieten. Im Gegensatz zu Österreich (17 Milchkühe pro Betrieb), werden in Deutschland durchschnittlich rund 64 Tiere pro Betrieb gehalten. In beiden Ländern wird ein deutliches Größenwachstum der Viehbestände beobachtet (VÖM, 2016; BMLFUW, 2017; MIV, 2017; Statistisches Bundesamt, 2018).

- Mit 31,3 Mio. Tonnen wurde 2016 in deutschen Molkereien etwa zehnmal mehr Milch verarbeitet als in Österreich (3,2 Mio. Tonnen). Deutschland nimmt damit europaweit den Spitzenplatz ein (BMLFUW, 2016; BMEL, 2017).

- Auch beim Umsatz liegt die deutsche Molkereibranche 2016 mit 21,9 Mrd. Euro etwa zehnmal höher als Österreich (2,45 Mrd. Euro). So erwirtschaftete die größte Molkerei Deutschlands 2016 einen Umsatz von 4,6 Mrd. Euro, wobei alleine der Umsatz der siebtgrößten Molkerei in Deutschland (Zott mit 902 Mio. Euro) weit über dem der größten österreichischen Molkerei (Berglandmilch mit 810 Mio. Euro) lag (MIV, 2016; BMLFUW, 2017).

- Eine Besonderheit in Österreich und Deutschland stellt die überwiegend genossenschaftliche Struktur vieler milchverarbeitenden Unternehmen dar, die Landwirte 
sind hier auch Inhaber der Molkereien (Tribl und Salhofer, 2013). In Österreich liegt der Anteil der Genossenschaftsmolkereien bei 95 Prozent; in Deutschland bei 65 Prozent (BMi, 2014).

- Die mengenbezogenen Hauptprodukte in deutschen und österreichischen Molkereien sind das Segment „weiße Produkte" mit Trinkmilch und Joghurt oder Sahneerzeugnisse sowie Käse. Den höchsten Wertanteil erzielen beide Länder mit Käse (MIV, 2017; AMA, 2019).

Österreicher konsumieren pro Kopf durchschnittlich 76,6 Kilogramm Konsummilch und 20,6 Kilogramm Käse (BMLFUW, 2016). In Deutschland lag der durchschnittliche Konsum von Konsummilch im Jahr 2017 bei insgesamt $51,5 \mathrm{~kg}$ und jener von Käse bei 24,1 kg pro Person. Beide Länder weisen im Vergleich zu den Vorjahren eine kontinuierliche Steigerung bei Käse auf, wohingegen ein Rückgang bei Kondensmilch, Sahneerzeugnissen und Butter zu beobachten ist (BMLFUW, 2016; BMEL, 2017). Das hohe Ansehen der Molkereibranche (Albersmeier und Spiller, 2009) ist nicht zuletzt auf das positiv besetzte Image von Milch zurückzuführen; Milch wird von der Mehrheit der Verbraucher mit Gesundheit und Vitalität in Verbindung gebracht. Darüber hinaus gelten Milch und Milchprodukte als wichtiger Bestandteil einer ausgewogenen Ernährung (Wienert, 2007). Dem gegenüber rücken kritische Stimmen zu Tierhaltung, Produktionsbedingungen und Gesundheitswerten der Produkte ebenso in das öffentliche Bewusstsein, wie ökonomische Themen, insbesondere die „Milchpreisentwicklung“ (Wienert, 2007; Nieberg und Schleenbecker, 2014; Forsa, 2017; MIV, 2017).

Die Herausforderungen des Marktes, den qualitätsbezogenen Wünschen des Handels sowie der Konsumenten begegnet die Milchbranche in beiden Ländern mit entsprechenden Produktinnovationen bzw. -qualitäten. Die österreichischen Molkereien verarbeiten bereits seit 2010 ausschließlich zertifiziert gentechnikfreie Milch und nehmen somit international eine Vorreiterrolle ein (ARGE Gentechnik-frei, 2015). Davon wurden im Jahr 2016 17 Prozent biologische Milch und 13 Prozent Heumilch an die Molkereien geliefert (BMLFUW, 2017). Letztere wurde im Jahr 2016 von der Europäischen Union (EU) mit dem g.t.S.-Siegel (garantiert traditionelle Spezialität) ausgezeichnet (VÖM, 2016). Beide Milcharten werden unter dem Begriff „Spezialmilch“ - ein Schwerpunkt der österreichischen Milchverarbeitung - zusammengefasst (BMLFUW, 2017). Deutschland produziert mit zwei Prozent biologischer Milch im Jahr 2015 nur einen geringen
Teil an Spezialmilch (MIV, 2017). Demgegenüber wächst der Anteil an gentechnikfreier Milch in Deutschland dynamisch: Waren es im Jahr 2011 noch 3 Prozent, meldete die Agrarmarkt-Informations-Gesellschaft (AMI) im Jahr 2017 bereits einen Anteil von 40 Prozent (Keunecke, 2017). Darüber hinaus fordert das BMEL (2017), eine insgesamt noch stärkere strategische Ausrichtung der deutschen Milchwirtschaft auf Qualitätsführerschaft bei Milcherzeugnissen und damit eine deutlichere Ausrichtung auf Verbraucherwünsche.

\section{Theoretische Grundlagen}

Das grundlegende Konzept der Exzellenz in Unternehmen und dessen Parameter wird im folgenden Teilkapitel 4.1. beschrieben und darin die Themenfelder "Prämierungen“ (Kapitelteil 4.2.), „Zertifizierungen“ (Kapitelteil 4.3.) sowie „Nachhaltigkeitskommunikation“ (Kapitelteil 4.3.) verortet. Dieser Zugang dient als Basis für die weitere Beurteilung von Exzellenzperformance und Exzellenzkommunikation in der Milchwirtschaft in Österreich und Deutschland sowie den darauf aufbauenden Ländervergleich.

\subsection{Exzellenz in Unternehmen}

Exzellenz kennzeichnet sich durch eine herausragende Position oder hervorragende Leistungen, die besser - im Sinn von erfolgreicher - als im Mitwettbewerb realisiert werden. Demnach ist Exzellenz in zweifacher Hinsicht relativ: zum einen da Ansprüche, die das Exzellenzniveau bestimmen, sich nach ihrem Zielhorizont unterscheiden bzw. im Zeitverlauf ändern können; zum anderen, da sich die Abgrenzung immer auf das Bessersein im Vergleich zu anderen, den „bloß Simplen“ (Imhof, 2009) bezieht. Der Bezug auf ein Referenzniveau oder auf die Leistung bzw. Nicht-Leistung anderer ist notwendig, um Exzellenz sichtbar zu machen (Cronin und Gudim, 1987; Woywode, 2004; Johnson, 2006; Gebhardt, 2016). Dies bedeutet, exzellente Unternehmenstätigkeit, die Performance, alleine genügt nicht: $\mathrm{Um}$ sich als innovatives und erfolgreiches Unternehmen abzuheben, ist die Kommunikation einer solchen Exzellenz unerlässlich (Brunner und Wagner, 2011; Mast, 2013). Diese beiden Ebenen „Exzellenz-Performance“ und „Exzellenz-Kommunikation“ (Gebhardt et al., 2019), sind in Abbildung 1 zusammengefasst und werden in den folgenden Ausführungen nacheinander beschrieben. 
Exzellenz-Performance: In verschiedenen Modellen der organisationalen Exzellenz, auch Business-ExcellenceModells (BEM) genannt, werden deren Bausteine und Einflussfaktoren kategorisiert (Dahlgaard-Park und Dahlgaard, 2006). Vereinfacht beruht Exzellenz demnach auf den Menschen, die im Unternehmen arbeiten (People), dem Beachten der Kundenwünsche (Customers), den ständigen Innovationen (Innovation) sowie - als Basis von allem - dem Management solcher Veränderung in allen Unternehmensbereichen (Leadership). Hieraus leiten die Autoren einen Ansatz, das 4P-Modell der Unternehmensexzellenz ab, der die Felder 1) Management \& Prozesse, 2) Mitarbeiter (people), 3) Partnerschaften, 4) Produkte umfasst. Diese Exzellenzfaktoren bauen aufeinander auf und bedingen sich gegenseitig. Exzellenzstrategien können auf Abnehmer oder auf die Konkurrenz gerichtet sein und dabei Innovations-, Qualitäts-, Marken- und Kostenorientierung umfassen (Wunder und Bausch, 2014; Meffert et al., 2018) oder Kooperationen, Nischen- oder andere Differenzierungsstrategien (Dahlgaard-Park und Dahlgaard, 2006; Wunder und Bausch, 2015). In der Molkereibranche gelten vor allem Produktinnovationen, außerdem die Produktionstechnologie, der organisatorische Aufbau und das
Engagement der Führungsebene (Benes und Groh, 2012) sowie die Qualifikation der Mitarbeiter und Mitarbeiterinnen (Winkelmann, 2004) als signifikante Erfolgsfaktoren. In Gebhardt et al. (2019) werden Exzellenzfaktoren in der Ernährungswirtschaft zusammenfassend beschrieben.

Exzellenz-Kommunikation: Die kommunikative Ebene von Exzellenz bezieht sich auf die interne oder externe Darstellung der organisationalen Exzellenz. Über Homepages, in Unternehmens- oder Nachhaltigkeitsberichten bzw. mittels Werbung können Unternehmen selbstgewählt über sich und ihr besonderes Engagement in bestimmten Bereichen kommunizieren (Selbstberichte). Der eigene Internetauftritt zählt zu den am häufigsten genutzten Instrumenten von Unternehmen, vor allem in deren Nachhaltigkeitskommunikation, um ihre Zielgruppe wirkungsvoll zu erreichen (Gebhardt und Fülle, 2018), auch in der Ernährungswirtschaft (Gebhardt, 2016). Problematisch ist, dass Unternehmen dazu tendieren, eher positive als negative Informationen zu veröffentlichen (Hahn und Lülfs, 2014). Die Überprüfung durch neutrale Dritte, einer „Third-Party“, und hierauf basierende Zertifikate, Prüfsiegel oder Berichte (Drittberichte), entsprechen den vertrauensbildenden Instrumenten der

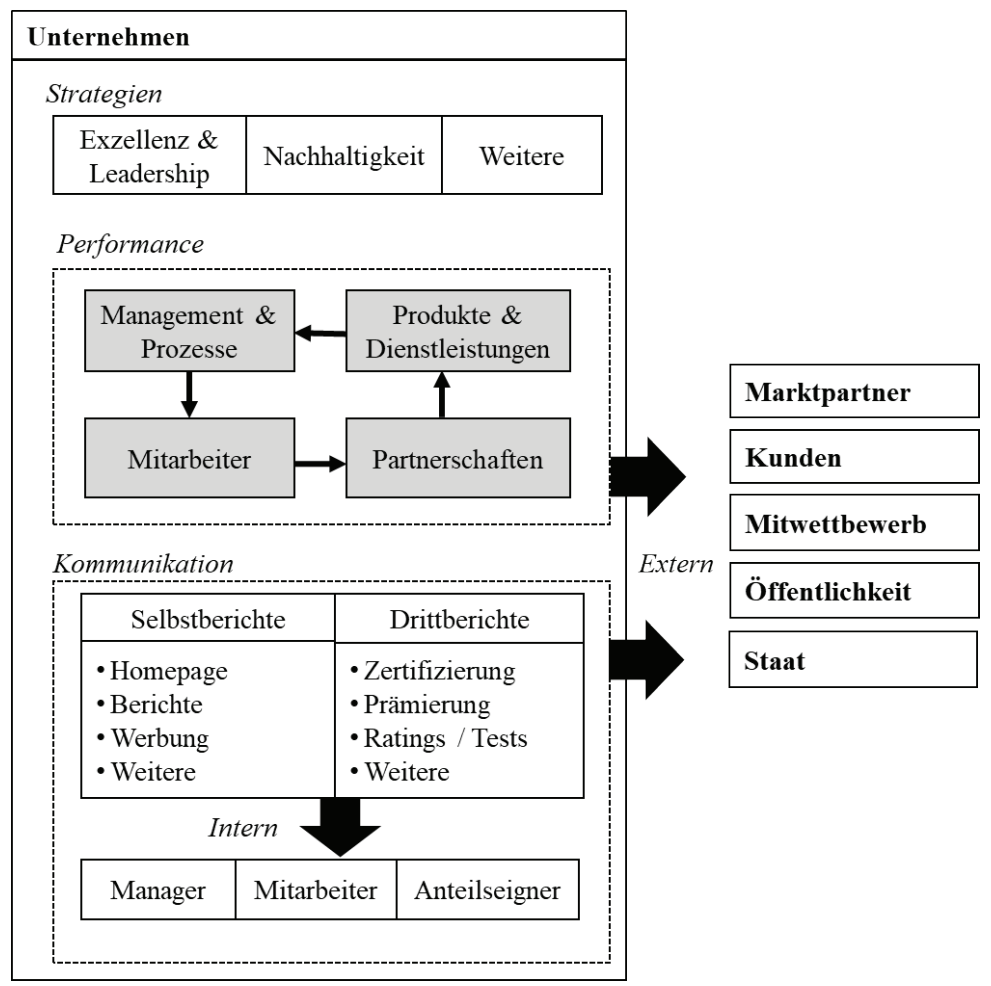

Abbildung 1. Exzellenz in Unternehmen im Zusammenspiel von Performance und Kommunikation. Quelle: Eigene Darstellung in Anlehnung an Gebhardt et al. (2019), Dahlgaard-Park und Dahlgaard (2006), Mast (2013)

Figure 1. Corpororate Excellence in the interaction of performance and communication 
Unternehmenskommunikation, die Informationsasymmetrien überwinden sollen (Mast, 2013). Eine herausragende Bewertung in Ratings und Tests oder eine gute Platzierung in Rankings führt außerdem zu Aufmerksamkeit unter den Geschäftspartnern sowie in der Gesellschaft (Mast, 2013). Mit dem Bedeutungszuwachs von Prämierungen steht den Unternehmen eine weitere Möglichkeit offen, sich fremdevaluiert als Vorreiter, „Bester“ oder "Sieger“ zu präsentieren (Gebhardt, 2016). Nach Gebhardt et al. (2019) stellt die Unternehmensgröße eine zentrale Einflussgröße der Exzellenzkommunikation in der Ernährungswirtschaft in Deutschland dar: Je größer ein Unternehmen, desto mehr wird über Nachhaltigkeit kommuniziert; desto vielfältiger und häufiger sind Zertifizierungen auf den Internetseiten zu finden. Gleiches gilt eingeschränkt für Prämierungen; über Produktprämierungen berichten auch viele kleinere Unternehmen.

\subsection{Prämierungen}

Von externen Stellen vergebene Prämierungen (engl. awards) sind ein anerkanntes und immer häufiger verliehenes Signal, um Unternehmen öffentlichkeitswirksam für ihre hervorragenden Leistungen auszuzeichnen. Mit Prämierungen soll besonderes Engagement belohnt, Vorbilder hervorgehoben und zum Nachahmen oder zu weiteren Verbesserungen angeregt werden (Frey und Gallus, 2014; Gebhardt, 2016). Die quantitative Limitierung ist charakterisierend für Awards und Voraussetzung für ihre Exklusivität. Es wird meist nur ein Sieger bzw. eine Siegerin oder einige wenige als Gewinner bzw. Gewinnerinnen im Rahmen eines Wettbewerbs ausgezeichnet. Prämierungen können nach dem Themenbezug (z. B. Nachhaltigkeit oder Innovationsgeist) sowie nach der Ausrichtung auf Produkt-, Projekt- oder Gesamtunternehmensebene unterschieden werden (Gebhardt, 2016). Beispielhaft sind hier bekannte Nachhaltigkeitsprämierungen wie der Deutsche Nachhaltigkeitspreis in Deutschland und der TRIGOS in Österreich zu nennen. Beliebte Produktprämierungen für Lebensmittel, die die sensorische Produktqualität beurteilen, sind die DLG-Medaillen in Deutschland oder der Käsekaiser der AMA-Marketing in Österreich. Durch ihren kompetitiven Ansatz sowie der limitierten Zahl an Preisträgern heben sich Prämierungen von Zertifizierungen und anderen Bewertungssystemen wie Ratings ab (Gebhardt, 2016) und erzielen daher auch eine höhere Aufmerksamkeit. Darüber hinaus gelten Nachhaltigkeitsprämierungen als leicht verständliche Indikatoren, um die Komplexität von Nachhaltigkeit zu kommunizieren sowie sich als Unternehmen sichtbar gegenüber Geschäftspartnern, Mitarbeitern sowie Kunden als Vorbild für herausragende Nachhaltigkeitsleistungen zu positionieren (Gebhardt, 2016). Diese Positionierung ist von Bedeutung, um eine ökologische, ökonomische und soziale Entwicklung im Sinn der Nachhaltigkeit voranzutreiben (Kurz und Wild, 2015). Da es sich bei der Vergabe von Prämierungen um einen Vergleich innerhalb des Teilnehmerkreises eines jeweiligen Wettbewerbsjahres handelt, bewerten Prämierungen ausschließlich eine relative Exzellenz, deren Niveau je nach Zusammensetzung der Teilnehmer variieren kann (Gebhardt, 2016). Die Grenzen von Awards liegen außerdem in einer Überhäufung mit Auszeichnungen, da ihre Wirkung und Glaubwürdigkeit von ihrer Seltenheit, also Exklusivität, abhängen (Hansen und Weisbrod, 1972; Gebhardt, 2016).

\subsection{Zertifizierungen}

Zertifizierungen - sowie hierauf basierende Labels - stellen eine weitere Form der Auszeichnung besonderer Leistungen dar (Gebhardt, 2016), bei denen jedoch die quantitative Dimension grundsätzlich unbeschränkt bleibt und die Auswahl alleine von der Erfüllung vorgegebener Standards abhängt. Deren Überschreiten ist meist grundlegend, um in den Zirkel der Ausgewählten aufgenommen zu werden und damit eine vergleichsweise exklusive Stellung einzunehmen. Demgegenüber führt das Nicht-Erfüllen bzw. Unterschreiten zur vollständigen Nicht-Akkreditierung des Akteurs; dieser bleibt vom Kreis der Ausgezeichneten ausgeschlossen (Gebhardt, 2016). Weiters betreffen die gesetzlich vorgeschriebenen oder freiwilligen Zertifikate verschiedene Unternehmensbereiche. Zander (2016) kategorisiert Zertifikate nach ihren Zielgruppen. Zertifikate im Bereich „Business to Busines" (B2B) ermöglichen den Marktzugang und erleichtern Kooperationen. Solche Zertifikate betreffend Qualitätsmanagement (beispielsweise aus der ISO-Normenfamilie) oder den LEH (beispielsweise International Featured Standard Food - IFS) bleiben für Konsumenten meist verborgen. Demgegenüber stehen Zertifikate im Bereich „Business to Consumer“ (B2C), welche sich für die Verbraucherkommunikation eignen. Sie stehen für Informationen bezüglich Qualität, Produktionsweise oder Verarbeitung. Dazu zählen Zertifikate zur biologischen oder gentechnikfreien Produktionsweise sowie Nachhaltigkeit (Pöchtrager, 2011; Meyer-Höfer und Spiller, 2016; Zander, 2016). Zertifikate bestätigen zudem 
die Einhaltung bestimmter Standards (Meyer-Höfer und Spiller, 2016), deren Benchmarks auch für verschiedene Qualitätsstufen festgelegt sein können (z. B. TierschutzLabels in Deutschland). Bei anderen Zertifikaten reicht es aus, dass bestimmte als unkritisch bezeichnete Standards teilweise oder erst zu einem späteren Zeitpunkt erfüllt sind (z. B. Rainforest Alliance) oder alleine eine Selbstverpflichtungserklärung abgegeben wird, vorgegebene Leitsätze einhalten zu wollen (Gebhardt, 2016). Töpfer (2006) kritisiert außerdem, dass für Zertifikate im Qualitätsmanagement (z. B. ISO 9000) Abläufe primär dokumentiert werden - dabei fehle neben der Optimierung auch der Bezug zu Kundenanforderungen.

\subsection{Nachhaltigkeit und Kommunikation}

Gemäß dem Drei-Säulen-Modell umfasst Nachhaltigkeit ökologische, ökonomische und soziale Dimensionen (Pufé, 2014). Als strategischer Erfolgsfaktor kann ein langfristig ausgerichtetes, wertegenerierendes Nachhaltigkeitsengagement auch zur Differenzierung von Mitbewerbern beitragen (Kirchhoff, 2006; Hutton und Mayer, 2010). Als erfolgsentscheidend gelten dabei neben einer nach innen und außen gerichteten Übernahme gesellschaftlicher Verantwortung auf Führungsebene (CSR) (Lendle, 2012) auch eine transparente und vertrauensgenerierende Nachhaltigkeitskommunikation gegenüber internen und externen Stakeholdern (Brugger, 2010; Friedrich et al., 2013; Kurz und Wild, 2015). Obwohl die Kommunikation über Nachhaltigkeit in der Ernährungswirtschaft für Unternehmen aller Größen eine hohe Relevanz besitzt, weist sie eine nur geringe Vielfalt hinsichtlich Inhaltes und Form auf. Die Milchwirtschaft in Deutschland ordnet sich hier im Vergleich zu anderen Lebensmittelbranchen im Mittelfeld ein (ZNU, 2013; Rottwild und Theuvsen, 2016). Gefolgt von Geschäftsberichten, Pressemitteilungen sowie Produktkennzeichnungen stellt der eigene Internetauftritt für Unternehmen der Ernährungswirtschaft das wichtigste Instrument dar, um wirkungsvoll ihre Zielgruppen mit Nachhaltigkeitsthemen zu erreichen (Gebhardt, 2016). Besondere Nachhaltigkeitsleistungen können zudem durch die Veröffentlichung von Nachhaltigkeitsberichten kommuniziert werden, die zunehmend das, primär auf ökologische Belange ausgerichtete Instrument der Umweltberichterstattung ablösen. In Deutschland gilt seit 2018 eine nichtfinanzielle Berichtspflicht (Nachhaltigkeitsbericht) für große Unternehmen (RNE, 2014). Die Global Reporting Initiative (GRI, 2015) gibt internationale Richtlinien für die Erstellung von ganzheitlichen Nachhaltigkeitsberichten vor, die für mehr Transparenz und Vertrauen sorgen sollen. Zudem können herausragende Nachhaltigkeitsleistungen mit Prämierungen hervorgehoben oder mit Zertifizierungen bestätigt werden (Europäische Kommission, 2011; Friedrich et al., 2013; Gebhardt, 2016).

Insofern können, die in der Inhaltsanalyse in Kapitel 5 und 6 berücksichtigten Formen der Exzellenzkommunikation in unterschiedlich hohem Maße Exzellenz bezeugen: Prämierungen vor Zertifizierungen, Zertifizierungen vor Selbstberichten (Gebhardt, 2016).

\section{Material und Methodik}

Zur Darstellung von Exzellenz in der Molkereibranche orientiert sich die vorliegende Studie an einem zweistufigen Ansatz von Gebhardt et al. (2019), welcher die Ebene der Unternehmensführung, hier die Exzellenzenz-Performance (EP), und die Ebene der Unternehmenskommunikation, hier die Exzellenzkommunikation (EK), umfasst. Diese beiden Ebenen werden in einem Ländervergleich Österreich und Deutschland gegenübergestellt. Dafür wurden länderbezogene Einzelstudien durchgeführt, die jeweils zweistufig aufgebaut sind: Einer Expertenbefragung folgte die Analyse der Internetdarstellung (Website) ausgewählter Unternehmen. Insgesamt 22 Experteninterviews und 197 Datensätze aus der Website-Analyse stehen der Analyse zur Verfügung (vgl. Tabelle 1). Die Kategorisierung der Molkereien folgt der Empfehlung der Europäischen Kommission (2003), wonach Klein- sowie Kleinstunternehmen mit bis zu 49 Mitarbeitern („klein“) und mittlere Unternehmen mit 50 bis 249 Mitarbeitern („mittel“) kennzeichnen. Unternehmen mit über 250 Mitarbeitern werden den Großunternehmen („groß“) zugeordnet. Zudem erfolgte eine weitere Kategorisierung zur Produktionsausrichtung hinsichtlich Biolebensmittel.

\subsection{Experteninterviews in Österreich und Deutschland}

In Österreich wurden im Frühjahr 2017 insgesamt neun Face-to-Face-Interviews mit Vertretern von je drei großen, mittleren und kleinen Molkereien geführt. Bei der Fallauswahl wurden primär die Unternehmensgröße und sekundär unterschiedliche Rechtsformen und Produktionsausrichtungen hinsichtlich Bio-Lebensmittel berücksichtigt. Die Interviewdauer betrug 45 bis 60 Minuten. Die Auswertung der Transkripte erfolgte mittels qualitativer Inhaltsanalyse 
Tabelle 1. Übersicht der befragten Experten und Stichprobe der Website-Analyse

Table 1. Overview of the surveyed experts and the sample of the website analysis

\begin{tabular}{|c|c|c|c|c|c|c|}
\hline & \multirow[b]{2}{*}{ Anzahl } & \multirow{2}{*}{$\begin{array}{l}\text { Gesamt } \\
\text { Prozent }\end{array}$} & & \multirow{2}{*}{$\begin{array}{r}\text { Österreich }(\mathbf{O}) \\
\text { Prozent }\end{array}$} & \multicolumn{2}{|c|}{ Deutschland (D) } \\
\hline & & & Anzahl & & Anzahl & Prozent \\
\hline \multicolumn{7}{|l|}{ Experteninterviews } \\
\hline gesamt & 22 & 100 & 9 & 100 & 13 & 100 \\
\hline mit Molkereivertretern & 21 & 95 & 9 & 100 & 12 & 92 \\
\hline mit Unternehmensberatern & 1 & 5 & 0 & 0 & 1 & 8 \\
\hline \multicolumn{7}{|l|}{ Website-Analyse } \\
\hline gesamt & 197 & 100 & 63 & 100 & 134 & 100 \\
\hline \multicolumn{7}{|l|}{ nach Unternehmensgröße } \\
\hline klein & 118 & 60 & 50 & 79 & 68 & 51 \\
\hline mittel & 45 & 23 & 7 & 11 & 38 & 28 \\
\hline groß & 34 & 17 & 6 & 10 & 28 & 21 \\
\hline \multicolumn{7}{|l|}{ nach Produktionsausrichtung } \\
\hline nur konventionell & 58 & 29 & 25 & 41 & 33 & 25 \\
\hline $\begin{array}{r}\text { gemischt } \\
(\text { bio }+ \text { konventionell })\end{array}$ & 87 & 44 & 20 & 32 & 78 & 58 \\
\hline nur bio & 40 & 20 & 17 & 27 & 23 & 17 \\
\hline
\end{tabular}

nach Mayring (2015) auf Basis eines Kategoriensystems, das sich an den fünf Themenfeldern des Gesprächsleitfadens (Verständnis von Exzellenz; Darstellung von Exzellenz; Bedeutung von Nachhaltigkeit; Bedeutung von Prämierungen; Bedeutung von Zertifizierungen) anknüpft. Um die länderübergreifende Vergleichbarkeit sicherzustellen, orientierte sich der Aufbau des Interviewleitfadens an der deutschen Expertenbefragung, bei der im Frühjahr 2016 insgesamt 13 Telefoninterviews, davon 12 mit Vertretern der Milchwirtschaft unterschiedlicher Größe (zwei große, je fünf mittlere und kleine Unternehmen) und eines mit einem Unternehmensberater geführt wurde. Die Auswertung erfolgte ebenfalls mittels qualitativer Inhaltsanalyse nach Mayring (2015) auf Kategorienebene.

\subsection{Website-Analysen österreichischer und deutscher Molkereien}

Ergänzend erfolgte in beiden Ländern eine inhaltsanalytische Untersuchung von Molkerei-Internetpräsenzen hinsichtlich der Kommunikation über Nachhaltigkeit sowie über Prämierungen und Zertifizierungen, wobei die erhobenen Parameter in beiden Studien weitgehend deckungsgleich waren. Generell wurden ausschließlich Unternehmen analysiert, welche vorab definierte Kriterien (z. B. eigene Website) erfüllten. Tabelle 1 zeigt eine
Kategorisierung der untersuchten Molkereien hinsichtlich ihrer Größe und Produktionsausrichtung.

Basierend auf einer Branchenabfrage bei der Unternehmensdatenbank AURELIA zu „Milchverarbeitung (ohne Herstellung von Speiseeis)“ wurden am 2. Mai 2017 in Österreich 63 Molkereien erfasst. Die Erhebung schloss die Parameter Unternehmensbezeichnung, Rechtsform, Bundesland, Adresse sowie Anzahl der Mitarbeiter mit ein. Molkereien mit mehreren Betriebsstätten wurden dabei zusammengefasst. Die Internetseiten der $63 \mathrm{Mol}-$ kereien wurden außerdem anhand von zehn Kriterien zur Nachhaltigkeitskommunikation (Informationen über zumindest eine Säule der Nachhaltigkeit auf der Internetseite, Nachhaltigkeitsbericht), zu Prämierungen (ProduktAwards, Nachhaltigkeits-Awards oder sonstige betriebliche Prämierungen) sowie zu Zertifizierungen (ISO 9001, ISO 50001, ISO 14001, EMAS, Bio) untersucht und deskriptiv ausgewertet.

In Deutschland wurden im Mai 2016 in gleicher Weise Internetseiten von 134 milchverarbeitenden Betrieben aus den fünf Bundesländern Baden-Württemberg, Bayern, Berlin und Brandenburg sowie Schleswig-Holstein untersucht. Sofern die betrachteten Unternehmen gemäß der Verordnung (EG) Nr. 853/2004 „Verordnung über Meldepflichten über Marktordnungswaren " (MarktOW MeldV) als ein für den Handel mit Lebensmitteln 
tierischen Ursprungs in Deutschland zugelassener Betrieb in der Unterliste IX „Rohmilch und Molkereiprodukte“ veröffentlicht wurden, wurden sie in Anlehnung an den Strukturbericht (BMELV, 2008) und die Klassifikation der Wirtschaftszweige (Statistisches Bundesamt, 2008) ausgewählt. Unternehmen, die alleine Rohmilch oder Milchprodukte zum Direktverzehr bzw. zur Selbstvermarktung erzeugen, wurden dabei nicht berücksichtigt.

\subsection{Vorgehen des Ländervergleiches Österreich und Deutschland}

Für den Ländervergleich wurden die qualitativen Ergebnisse der Experteninterviews aus den beiden Einzelstudien in Österreich und Deutschland erneut hinsichtlich Gemeinsamkeiten und Unterschiede betrachtet. Dies erfolgte auf Kategorienebene sowie auf Basis der für diese Studie definierten Forschungsfragen. Die Ergebnisse der Website-Analysen für Österreich und Deutschland wurden in einer Frequenzanalyse in prozentualen Angaben einander gegenübergestellt.

\section{Ergebnisse}

Auf Basis der Literatur und den forschungsleitenden Fragestellungen analysiert die vorliegende Studie Exzellenz in der österreichischen und deutschen Molkereibranche in vier Kategorien, die die nachfolgenden Abschnitte 6.1 bis 6.4 betiteln. Die Ergebnisse der länderbezogenen Studien werden hierzu aus den Experteninterviews präsentiert und mit beispielgebenden Statements unterlegt. Zur Identifizierung der Experten wurde ihrer laufenden Nummerierung ein Ländercode (Ö für Österreich; D für Deutschland) vorangestellt. Zusammenfassend werden im Kapitelteil 6.5. die Ergebnisse der Website-Analyse im Ländervergleich gegenübergestellt.

\subsection{Bedeutung und Verständnis von Exzellenz und deren Kernbereiche}

Exzellenz hat für die milchverarbeitende Wirtschaft eine hohe und auch weiter zunehmende Bedeutung, so die gleichlautenden Ergebnisse der Expertenbefragungen in Österreich und Deutschland. Globalisierung, Strukturwandel und Wegfall der Milchquote sowie die steigende Marktsättigung werden in beiden Ländern als die starken Treiber für die Notwendigkeit von Exzellenz in der Milch- wirtschaft benannt. Die österreichischen Experten beobachten insbesondere seit dem EU-Beitritt Österreichs im Jahr 1995 Veränderungen am Markt, weswegen das Thema „Exzellenz“ für sie verstärkt an Bedeutung gewinnt.

Exzellenz wird in beiden Ländern als ein „Bessersein“ oder „Herausstechen“ im Vergleich zur Konkurrenz beschrieben. Dies bezieht sich vor allem auf hervorragende Produkte und Leistungen, die besser, im Sinne von erfolgreicher oder einzigartiger, als von den Mitwettbewerbern in der Milchbranche realisiert werden. Die Berücksichtigung von Kundenwünschen und gesellschaftlichen Erwartungen seien hierbei essenziell. Was unter Exzellenz zu verstehen ist, fasst ein/e österreichische/r Expert/in wie folgt zusammen: „Exzellenz beginnt immer dort, wo man etwas viel besser macht als andere" (Ö-E9). Ein/e deutsche/r Bio-Erzeuger/in beschreibt Exzellenz als ein Mittel, um „sich nicht so vergleichbar zu machen" (D-E5). Dieses einzigartige Leistungsversprechen und Alleinstellungsmerkmal (engl. Unique-Selling-Proposition (USP)) herauszustellen, stellt in der hoch kompetitiven Milchbranche und dem Milchmarkt mit vielen ähnlichen Produkten eine besondere Herausforderung für die Unternehmen beider Länder dar.

Exzellenz wird als vielschichtig beschrieben: Sie bezieht sich auf die funktionelle, technische, ästhetische und ethische Produktgestaltung, den Rohstoffen sowie auf das Unternehmen selbst, dem „Gesamtpaket" (D-E7). Aus diesem Verständnis resultieren sechs zentrale Kernbereiche, welche die Exzellenz von milchverarbeitenden Unternehmen umfasst: Produkte, Rohstoffe, Management \& Prozesse, Mitarbeiter, Kunden sowie alle weiteren Partnerschaften, vor allem mit den Landwirten sowie die gesellschaftliche Verantwortung (vgl. Tabelle 2). Dies beinhaltet die vier Grundelemente des in Kapitel 4 beschriebenen ExzellenzModells von Dahlgaard-Park und Dahlgaard (2006) und erweitert es um die drei Bereiche: Rohstoffe für Molkereiprodukte, Lieferantenbeziehung sowie gesellschaftliche Verantwortung. Die Aussagen des Experten beider Länder stimmen hierbei überein, sie unterscheiden sich indes in Details. Dies ist in Tabelle 2 dargestellt. Im Weiteren werden zentrale Aussagen zu diesen Kernbereichen von Exzellenz und den länderbezogenen Unterschieden beschrieben. Erstklassige, einzigartige oder auch innovative Produkte mit einer herausragenden Rezeptur, die sich stark an den Kundenbedürfnissen orientieren und zudem den „Standard“ übertreffen - dies sind für die befragten Experten in Österreich und Deutschland zuvorderst Kennzeichen von Exzellenz einer Molkerei. Solchermaßen exzellente Molkereiprodukte zeichnen sich primär durch eine hochwertige 
Tabelle 2. Übersicht der Kernbereiche von Exzellenz in der Molkereibranche in Österreich und Deutschland

Table 2. Overview of the areas of excellence in the dairy industry in Austria and Germany

\begin{tabular}{|c|c|c|c|}
\hline Exzellenzbereiche & Gemeinsame Nennungen & Exklusiv Ö & Exklusiv D \\
\hline Produkte & $\begin{array}{l}\text { Herausragende, bestmögliche Qualität \& Rezeptur } \\
\text { Einzigartige Produkte } \\
\text { Produktvielfalt \& -abwechslung } \\
\text { Produktsicherheit } \\
\text { Authentische Produkte } \\
\text { Markenbildung \& starke Marken }\end{array}$ & $\begin{array}{l}\text { Innovative Produkte } \\
\text { Produkthygiene }\end{array}$ & $\begin{array}{l}\text { Naturnahe \& natürliche Produkte } \\
\text { Aus schonenden Verfahren } \\
\text { Verpackung }\end{array}$ \\
\hline Rohstoffe & $\begin{array}{l}\text { Bestmögliche Qualität der Zutaten \& des Futters } \\
\text { Herkunft der Zutaten } \\
\text { Tiergesundheit \& Tierwohl }\end{array}$ & Herkunft des Futters & Artgerechte Tierhaltung \\
\hline Management \& Prozesse & $\begin{array}{l}\text { Langfristigkeit \& Dauerhaftigkeit } \\
\text { Innovationsfähigkeit \& neue Technologien } \\
\text { Anpassungsbereitschaft \& } \\
\text {-fähigkeit } \\
\text { Spezialisierung \& Differenzierung } \\
\text { Erfolgsorientierung \& Wachstum } \\
\text { Ausstattung \& Gebäude }\end{array}$ & $\begin{array}{l}\text { Etabliertheit } \\
\text { Standort } \\
\text { Vorbildrolle }\end{array}$ & Neue Märkte \\
\hline Mitarbeiter & $\begin{array}{l}\text { Kompetenz \& Erfahrung } \\
\text { Engagement \& Motivation } \\
\text { Mitarbeiterschulungen } \\
\text { Unternehmenskultur \& Arbeitsklima }\end{array}$ & Partizipation & Gute Bezahlung \\
\hline Kunden & $\begin{array}{l}\text { Nähe zum Kunden (B2B/B2C) } \\
\text { Sichtbarkeit } \\
\text { Vollständige Erfüllung von Kundenwünschen } \\
\text { Reklamationsmanagement }\end{array}$ & $\begin{array}{l}\text { Schnelle Erfüllung von Kunden- } \\
\text { wünschen } \\
\text { Hoher Servicegrad }\end{array}$ & Kundenakzeptanz \\
\hline Lieferanten & $\begin{array}{l}\text { Nähe zu Erzeuger/innen } \\
\text { Faire Partnerschaften mit Landwirten und Landwir- } \\
\text { tinnen } \\
\text { Vertrauen \& Offenheit \& Respekt }\end{array}$ & Nähe zu Inhaber-Genossen & \\
\hline Gesellschaft & $\begin{array}{l}\text { Gesellschaftliche Verantwortung } \\
\text { Nachhaltigkeit }\end{array}$ & & \\
\hline
\end{tabular}

Produktqualität mit bestmöglichen, hochwertigen Zutaten aus - die Produktqualitäten (Bio, Regional, Nachhaltigkeit und Tierwohl; zudem Authentizität, „Heumilch“ oder weitere Innovationen in Österreich; Natur und Natürlichkeit in Deutschland) sind es, mit denen Unternehmen sich spezialisieren oder Nischen besetzen. Zudem gelten die Einhaltung von Hygienerichtlinien und Produktsicherheit als wichtige Basisvoraussetzung für Milchprodukte. Exzellenz kann zudem auch im Massenmarkt umgesetzt werden, wenn „exzellente Produkte durch Pepp" (D-E13) und entsprechende Marktkommunikation erzeugt werden, wie ein/e Befragte/r in Deutschland berichtet. Exzellenz kann demnach als emotionsgeladener USP mittels Werbung, Verpackung oder Markenbildung dargestellt werden. Besondere Bedeutung hat dies für mittlere und große Unternehmen, die auf Wachstum und neue, auch internationale Märkte setzen.

Die Verwendung bestmöglicher Zutaten und Rohstoffe gelten als weiteres Zeichen von Exzellenz in der Molkerei- branche und werden in beiden Ländern mit der Futterqualität, dessen Herkunft sowie der Art der Tierhaltung (Stichwort: Weidehaltung) verbunden. In Österreich wird dabei die Herkunft des Futters hervorgehoben, in Deutschland eine artgerechte Tierhaltung.

Ebenso spielen der partnerschaftliche Umgang mit den Milchproduzenten. sowie ein fairer Milchpreis eine wichtige Rolle. Im Fall von kleinen und mittleren Genossenschaften - wie sie vor allem in Österreich anzutreffen sind - findet diese vertrauensvolle Nähe zu den genossenschaftlichen Inhabern, den Landwirten eine besondere Bedeutung. Die Experten beider Länder betonen außerdem, dass für die Herstellung exzellenter Produkte kompetente und motivierte Mitarbeiter unverzichtbar sind. Die Aus- und kontinuierliche Weiterbildung von Mitarbeitern sowie eine entsprechende Unternehmenskultur und ein gutes Arbeitsklima, um diese ans Unternehmen zu binden, sehen die Experten beider Länder als wesentlich für Exzellenz an. 
Aus österreichischer Sicht wird zudem die Partizipation der Mitarbeiter betont, aus deutscher Sicht eine gute Bezahlung. Darüber hinaus bilden die enge Zusammenarbeit mit den Geschäftskunden, den Endverbrauchern (vorausgesetzt diese gelten als Zielgruppe) sowie eine entsprechende Produkt- und Unternehmensdifferenzierung ein Kernstück von Exzellenzstrategien.

Exzellenz ist für Unternehmen kein finales Ergebnis, sondern Teil eines kontinuierlichen und demnach nie endenden Verbesserungsprozesses. Exzellenz ist eine veränderliche Größe, die vom permanenten Verbesserungsprozess in den Unternehmen und von den Gegebenheiten am Markt oder den Ansprüchen der Gesellschaft im Wandel der Zeit aufgehoben werden können: „Alles was du einmal eine Zeit lang gehabt hast ist Standard" (Ö-E9). Wenn der Mitwettbewerb nachzieht „ist die Exzellenz wieder dahin“ (Ö-E9). Es bleibt eine begrenzte Zeit, sich mit exzellenten Produkten zu positionieren. Dies gilt als Anreiz für Molkereien, frühzeitig und als Innovationsführer den Markt zu besetzen. Beispielhaft greifen die österreichischen Experten die 100 Prozent gentechnikfreie Milch auf, die seit 2010 in Österreich verpflichtend und damit am österreichischen Markt Standard ist. Dennoch beschreiben fast alle österreichischen Experten diese Spezialmilch als exzellent, vor allem mit Blick auf ausländische Märkte, insbesondere Deutschland. Nach Meinung der Experten steht dem Wunsch der deutschen Verbraucher nach gentechnikfreien Milcherzeugnissen zum Zeitpunkt der Befragung kein oder ein noch geringes Angebot von Seiten der deutschen Molkereien gegenüber (siehe Kapitel 3.1.). In beiden Ländern werden von Expertenseite damit einhergehend langfristiges Denken und Agieren, Anpassungsbereitschaft und -fähigkeit sowie Innovationsfähigkeit als wichtige Faktoren im Management und den betrieblichen Prozessen einer exzellenten Molkerei genannt. Weiters könnte dies in einigen Unternehmensbereichen bereits besser realisiert sein als in anderen. Darüber hinaus kann die Wahl eines „Mittelweges" (D-E7) - exzellente Produkte neben Standardprodukten im Portfolio zu haben - sowohl Erzeugern als auch Verarbeitern in der Milchbranche Stabilität bieten. Im Zusammenhang mit den immer stärker werdenden Handelsmarken (Ö-E9) stehen die österreichischen und deutschen Molkereien zudem vermehrt vor der Herausforderung, durch eine klare Produktdifferenzierung dem steigenden Konkurrenz- und Preisdruck entgegenzuwirken. Nicht zuletzt, da auch der LEH den Wert von Nachhaltigkeit für dessen Premium-Handelsmarken erkannt hat. Letztlich zielt die Exzellenz darauf ab, mehr Produkte (D-E9) zu ei- nem höheren Preis zu verkaufen, einen Regalplatz im Handel zu erhalten (Ö-E9) oder neue, internationale Märkte zu erschließen (D-E1).

\subsection{Die Bedeutung von Nachhaltigkeit als Teil von Exzellenz}

Das Verständnis von Exzellenz in der österreichischen als auch in der deutschen Molkereibranche ist untrennbar mit Nachhaltigkeit verbunden. Ein/e Vertreter/in einer österreichischen Großmolkerei sagt dazu: „Nachhaltigkeit ist eines der großen Themen, [...] um die Exzellenz eines Unternehmens voranzutreiben "(Ö-E9). Nachhaltigkeit stellt einen strategischer Erfolgsfaktor, aufgrund deren Komplexität aber auch eine kostenintensive Herausforderung dar, ebenfalls für große Unternehmen. Nachhaltigkeit wird gleichzeitig als ökonomisch notwendig erachtet und spielt für die Molkereibranche eine sehr große Rolle, „wenn wir auch zukünftig Geschäfte machen wollen" (D-E1). Das Nachhaltigkeitsengagement in der österreichischen und deutschen Molkereibranche nimmt zu, berichten die Befragten. Als Treiber eines nachhaltigen Engagements identifiziert die österreichische Molkereibranche vor allem den LEH, in Deutschland außerdem die Anforderungen ihrer Geschäftspartner in internationalen Märkten.

Obwohl der Begriff „Nachhaltigkeit“ ökonomische, ökologische und soziale Dimensionen verbindet, fehlt ein einheitliches Verständnis in der Branche. So steht primär die ökologische Dimension (z. B. Ressourceneinsparung, Umweltaspekt bei Bewirtschaftungsformen) im Vordergrund und wird teilweise synonym für die anderen Bereiche, insbesondere die soziale Nachhaltigkeit, verwendet. Soziale Nachhaltigkeit umfasst dabei beispielsweise den Umgang mit den Mitarbeitern oder die Partnerschaft mit den Produzenten. In diesen Zusammenhang wird Nachhaltigkeit vor allem für kleine Molkereien als Chance zur Differenzierung gegenüber dem steigenden Kosten- und Konkurrenzdruck der Branche gesehen.

Glaubwürdigkeit und Ehrlichkeit sowie Transparenz und Offenheit gelten laut den Experten als Erfolgsfaktoren der Kommunikation über ein entsprechendes Nachhaltigkeitsengagement. Diesen Anforderungen entsprechen am ehesten Nachhaltigkeitsberichte, welche standardisiert aufgebaut sind und somit vergleichbarere Informationen enthalten. Das Vertrauen der Kunden könne auch im Falle einer herausragenden Beurteilung ihrer Nachhaltigkeitsaktivitäten durch externe Institutionen steigen, berichten die befragten Molkereien. Aufgrund fehlender finanzieller 
und personeller Ressourcen stellt eine systematische Nachhaltigkeitskommunikation, insbesondere mittels Nachhaltigkeitsberichten, vor allem kleine Molkereien in beiden Ländern vor Herausforderungen. Dies zeigen im Kapitelteil 6.5. auch die Ergebnisse der Website-Analyse.

Nachhaltigkeit setzen vor allem große Molkereien in Deutschland gezielt für ihre Positionierung ein. Die befragten kleineren deutschen Molkereien sehen im Bio- bzw. Demeter-Aspekt positive Wirkungen einer ganzheitlichen Nachhaltigkeitskommunikation nach außen. Auch die österreichischen Experten berichten über das Bemühen österreichischer Molkereien, das Thema „Nachhaltigkeit“ für sich zu nutzen. Allerdings fehlt sowohl zwischen den Unternehmen als auch zwischen den Jahren die Vergleichbarkeit der Leistungen im Nachhaltigkeitsbereich. Die lückenhafte und unregelmäßige Kommunikation, als auch die allgemeine Informationsflut tragen dazu bei, dass die Leistungen von der Öffentlichkeit nur geringfügig wahrgenommen werden. Erfolge können hier mittels zielgruppenadäquater Informationsaufbereitung erreicht werden: Der Einsatz von E-Autos in der Molkerei oder die Offenlegung von Produktionsbedingungen und Herkunft der Milch (z. B. Heumilch g.t.S.) werden als Beispiele für Österreich angeführt. Experten aus Deutschland betonen außerdem die Kommunikation von Exzellenz und Nachhaltigkeit nach innen, um diese „im Unternehmen leben zu lassen" (D-E9).

\subsection{Bedeutung von Prämierungen (engl. awards) zur Darstellung von Exzellenz}

Um exzellent zu sein, gehört zwingend die Kommunikation darüber dazu. Es ist den befragten Unternehmen der Milchwirtschaft beider Länder ein Anliegen, ihre Exzellenz darzustellen und am Markt zu kommunizieren; manchmal vor der Maßgabe: „Wir wollen uns so gut wie möglich darstellen, ohne da etwas zu übertreiben" (D-E5). Diese Sorge aus Deutschland spiegelt die Uneinigkeit über die Bedeutung von Prämierungen zur Darstellung von Exzellenz und einer Vorbildrolle wider: Die Interviews zeigen, dass österreichische Molkereivertreter Prämierungen insgesamt wichtiger als deutsche einstufen. Ein/e österreichische/r Expert/in erklärt dies beispielhaft: „[...] weil man das Medienecho dahinter hat, im Gespräch bleibt und [...] immer wieder zeigt, dass man da ist und gut ist und dass man was Interessantes hat" (Ö-E2). Dies geht einher mit einer häufigeren Kommunikation über erhaltene Prämierungen von österreichischen Molkereien, wie die Website-Analyse zeigt (vgl. Kapitelteil 6.4.).
Die Expertenbefragung beider Länder verdeutlicht außerdem, dass gerade kleinere und mittlere Molkereien mit meist geringem Werbebudget (Ö-E1; Ö-E2) Prämierungen als Chance für ihre Kommunikation - vor allem auf ihrer Homepage oder über Social Media - sehen. Die Kosten einer Wettbewerbsteilnahme werden als eher gering eingestuft. Die Teilnahme erfolgt letztlich in Abhängigkeit individueller Zeit- und Budgetressourcen der Molkereien oder den Themen: „Neue Ideen, Technologien oder Produkte müssen Mitbewerber nicht sofort wissen "(Ö-E4).

Sowohl in Österreich als auch Deutschland gelten Produkt-, Unternehmens- sowie Nachhaltigkeitsprämierungen grundsätzlich als sichtbarer Beweis für eine überdurchschnittliche Leistungsqualität und damit als exzellentes Kommunikationsmittel. Speziell deutsche Molkereien priorisieren eine hohe Exklusivität bei Prämierungen. Einen positiven Effekt von Prämierungen auf das Image attestiert die deutsche Molkereibranche so letztlich in Abhängigkeit von Art und hoher Reputation der Vergabeinstitution, des Bewerbungsaufwandes und der Transparenz. Die Bedeutung von Prämierungen für Nachhaltigkeit und damit auch ihr Kommunikationswert steigen, wenn nicht nur einzelne Produkte, sondern dem holistischen Ansatz entsprechend, ganze Unternehmen ausgezeichnet werden. Die Wirkung von Prämierungen auf den Kaufentscheid bleibt indes generell ungeklärt.

Die aktuelle Entwicklung und - weiterhin erwartete - starke Zunahme von Wettbewerben werden in beiden Ländern kritisch betrachtet. Zur „Siegelflut" würden immer häufiger auch Prämierungen beitragen. Im Einzelnen wird in Deutschland die Deutsche-Landwirtschafts-Gesellschaft(DLG)-Medaille als Negativbeispiel wiederholt benannt: Da alle Produkte je nach Niveau der erreichten Punkteanzahl bei den sensorischen Qualitätsprüfungen eine goldene, silberne oder bronzene Medaille erhalten, führe dies zu einer entsprechend hohen Anzahl an Auszeichnungen und gehe zu Lasten der Exklusivität. In Österreich wird ebenfalls vor einem Bedeutungsverlust und einer Überforderung der Konsumenten gewarnt sowie mehr Exklusivität von Prämierungen und mehr Aufklärungsarbeit gefordert. Da nicht alle Molkereien an allen Wettbewerben teilnehmen, erlauben Prämierungen ohnehin nur die Auszeichnung der als besten bewerteten Leistung des jeweiligen Teilnehmer-Pools sowie den selbst ausgewählten Leistungen aus dem Produktportfolio der Teilnehmer, die in der Regel „nur das Beste und nicht die Standardversion"(Ö-E5) seien. 


\subsection{Bedeutung von Zertifizierungen zur Darstellung von Exzellenz}

Neben Prämierungen bieten auch Zertifikate für Unternehmen die Chance, ihre besonderen Leistungen zu zeigen. Da der LEH und die gewerblichen Kunden einen Großteil der mit Zertifikaten belegten Qualitätsansprüche vorgeben, erachten die befragten Experten in Österreich und Deutschland Zertifikate jedoch nur eingeschränkt als Zeichen für Exzellenz eines Unternehmens. Insbesondere bezieht sich dies auf Zertifikate wie der europäische IFSFood Standard, der zum Großteil vom LEH vorgeschrieben wird, sowie die eigenen Zertifizierungskataloge des LEH. Diese Zertifikate gelten als Grundstock und Voraussetzung für Exzellenz, ebenso wie weitere Zertifikate aus dem Qualitätsmanagement, „ohne dem [wir] gar nicht mehr die Erfolge haben könnten"(Ö-E6).

Hingegen entsprechen spezielle Produktionsweisen, wie biologisch, Heumilch oder gentechnikfrei, besonderen Verbraucherwünschen; sie werden als mögliche Themen gesehen, sich im Sinne von Exzellenz kommunikativ hervorzuheben und damit die Kundschaft zu erreichen. Die in Österreich seit 2010 flächendeckend als Standard angebotene gentechnikfreie Milch nimmt eine internationale Vorbildrolle ein. Daraus ergibt sich die Chance zur qualitativen Differenzierung österreichischer Molkereien in internationalen Märkten: Gentechnikfreie Produkte und Heumilch liegen vor allem im Ausland im Trend (siehe Kapitelteil 3.1.). Im österreichischen Inland hingegen „bringt das jetzt auch nicht mehr" (Ö-E5); das gesamte inländische Niveau der österreichischen Molkereien wurde auf diesen Standard angehoben (Ö-E2). In Österreich werden „Bio“, regionales Futter, Wiesen- oder Weidemilch, Bergbauernmilch sowie laktosefrei und entsprechende Zertifikate als neue oder wichtige Exzellenzfelder identifiziert. In Deutschland sind Clean-Label und der Nachweis natürlicher Attribute oder umweltfreundliche Verpackungen interessante Themen. Herausforderungen von Zertifizierungen werden in beiden Ländern im hohen Zeit- und Kostenaufwand, beispielsweise für Audits, festgehalten. Die Aufmerksamkeit der potenziellen Käufer müsse außerdem durch weitere Kommunikationsinstrumente und Werbemaßnahmen gewonnen werden; ein erhaltenes Zertifikat alleine reiche dafür nicht aus. Anders als erhaltene Prämierungen lassen sich Zertifizierungen beispielsweise kaum in Pressemitteilungen darstellen (D-E13). Zertifizierungen verdeutlichen außerdem den permanenten Druck im Verbesserungsprozess und der Exzellenzkommunikation, „man kann immer nur oben drauf [setzen], wieder eines und dann noch eines, [...] wenn man eines nicht hat, ist es schon eines zu wenig" (Ö-E6).

\subsection{Exzellenzkommunikation der Molkereibranche im Ländervergleich}

Die Kommunikation der Molkereibranche beider Länder über Nachhaltigkeit, Prämierungen und Zertifizierungen wurde in der Website-Analyse für zehn Elemente festgehalten und in Tabelle 2 gegenübergestellt. Die zentralen Aussagen aus den Experteninterviews und die länderbezogenen Unterschiede der dabei beschriebenen Exzellenzkommunikation werden dabei bestätigt.

Nachhaltigkeitskommunikation: Über zwei Drittel der österreichischen und die Hälfte der deutschen Molkereien berichten von ihrem nachhaltigen Engagement auf ihrer Internetseite (Ö: 68 Prozent, D: 50 Prozent). Demgegenüber berichten in Österreich nur drei Prozent und in Deutschland 11 Prozent der primär mittleren und großen Molkereien einen Nachhaltigkeitsbericht veröffentlicht zu haben.

Prämierungen: Insgesamt kommunizieren 54 Prozent der österreichischen und 42 Prozent der deutschen Molkereien über Prämierungen. Unterteilt nach den Kategorien überwiegen deutlich Produktprämierungen in beiden Ländern (Ö: 52 Prozent; D: 35 Prozent). Dies knüpft an der hohen Bedeutung der Produktqualität für Exzellenz aus Sicht der Molkereibranche sowie an der quantitativ nur selten limitierten Vergabe von Produktprämierungen an (siehe Kapitelteil 6.1.). Nachhaltigkeitsprämierungen werden in beiden Ländern am seltensten unter allen betrachteten Kategorien von Prämierungen ausgewiesen (Ö: 13 Prozent; D: 5 Prozent). Relevante Nachhaltigkeitsprämierungen sind beispielsweise klima:aktiv-Preis, TRIGOS, Klimablatt in Österreich und der Nachhaltigkeitspreis der Verbraucherinitiative oder der Nachhaltigkeitspreis „Mit gutem Gewissen" in Deutschland.

Zertifizierungen: Den hohen Stellenwert von Zertifikaten in der Molkereibranche zeigen die Zahlen beider Länder. Am häufigsten werden in Österreich und Deutschland Zertifikate zur Produktion von Bio-Lebensmittel kommuniziert: Über ein Zertifikat für biologische Produkte verfügen 59 Prozent der österreichischen und 41 Prozent der deutschen Molkereien. Kleine Molkereien kommunizieren Bio-Zertifikate dabei häufiger als große. In Deutschland sind es kleine und mittelgroße Unternehmen, die ausschließlich biologische Produkte herstellen; in Österreich sind es ausschließlich kleine Unternehmen. Des Weiteren 
Tabelle 2. Deskriptive Ergebnisse der Website-Analyse der Molkereibranche im Ländervergleich

Table 2. Descriptive results of the website analysis in the dairy industry in Austria and Germany

\begin{tabular}{|c|c|c|c|c|c|c|c|}
\hline & & \multicolumn{2}{|c|}{ Gesamt } & \multicolumn{2}{|l|}{ Ö } & \multicolumn{2}{|l|}{$\mathbf{D}$} \\
\hline & & Anzahl & $\%$ & Anzahl & $\%$ & Anzahl & $\%$ \\
\hline \multirow[t]{2}{*}{ Nachhaltigkeitskommunikation } & Internetseite & 110 & 56 & 43 & 68 & 67 & 50 \\
\hline & Nachhaltigkeitsberichte & 13 & 7 & 2 & 3 & 11 & 8 \\
\hline \multirow[t]{3}{*}{ Prämierungen } & Produktpreise & 80 & 41 & 33 & 52 & 47 & 35 \\
\hline & Sonstige betriebliche Preise & 30 & 15 & 13 & 21 & 17 & 13 \\
\hline & Nachhaltigkeitspreise & 15 & 8 & 8 & 13 & 7 & 5 \\
\hline \multirow[t]{5}{*}{ Zertifizierungen } & ISO $9001 \&$ andere (Qualitätsmanagement) & 71 & 36 & 19 & 30 & 52 & 39 \\
\hline & ISO 50001 (Energiemanagement) & 18 & 9 & 0 & 0 & 18 & 13 \\
\hline & ISO 14001 (Umweltmanagement) & 12 & 6 & 1 & 2 & 11 & 8 \\
\hline & EMAS & 9 & 5 & 1 & 2 & 8 & 6 \\
\hline & Bio & 92 & 47 & 37 & 59 & 55 & 41 \\
\hline
\end{tabular}

werden in beiden Ländern vielfach Zertifikate des Qualitätsmanagements (Ö: 36 Prozent; D: 39 Prozent) herausgestellt. Nachhaltigkeitszertifikate oder weitere Nachweise im sozial-ökologischen Bereich sind auf der Interpräsenz seltener zu finden. Dies gilt insbesondere für österreichische Molkereien, etwas häufiger liegen diese in Deutschland vor. Zertifikate des Energiemanagements nach ISO 50001 nannte dabei keine der österreichischen Molkereien auf ihrer Website (Ö: 0 Prozent; D: 13 Prozent). Über die internationalen Umweltmanagementnormen ISO 14001 berichten zwei Prozent der betrachteten österreichischen Molkereien (D: 6 Prozent), über EMAS und Ökoprofit ebenfalls 2 Prozent (D: 8 Prozent). Über sozialökologischen Zertifizierungen berichten in Deutschland absolut und relativ primär große Unternehmen.

\section{Diskussion}

\subsection{Diskussion der Resultate}

Zur Beantwortung der ersten Forschungsfrage, welche Faktoren Exzellenz aus Sicht von Unternehmen der Molkereibranche umfasst und inwieweit sich hierbei Nachbaltigkeit einordnet, kommt die vorliegende Studie zum Schluss, dass Exzellenz multifaktoriell zu verstehen ist und sich erst im Vergleich mit dem inländischen und ausländischen Mitwettbewerb sowie im Zeitverlauf feststellen lässt. Die unternehmensspezifische Sichtweise von Exzellenz hängt dabei von den jeweiligen Unternehmensschwerpunkten ab und ist zu unterscheiden u. a. nach B2B vs. B2C, Massenmarkt vs. Nischenmarkt oder Kuhmilch vs. Ziegenmilch.
Insgesamt zeigt die vorliegende Studie: Exzellent zu sein, ist in der Molkereibranche in Österreich und Deutschland ein großes und aktuelles Thema; Nachhaltigkeit spielt dabei eine wesentliche Rolle.

Die in anderen Studien in den Vordergrund gestellte Produktqualität von Milch und Milcherzeugnissen (z. B. in Winkelmann, 2004) ist zentral für den Unternehmenserfolg, reicht für dauerhaft herausragende Leistungen alleine jedoch nicht aus. Vielmehr handelt es sich bei Exzellenz in der Molkereibranche um einen ganzheitlichen Ansatz, welcher neben der Herstellung von besonderen Produkten, auch beste Rohstoffe, die faire Partnerschaft mit den Milchproduzenten, die Aus- und Weiterbildung der Mitarbeiter, die flexible und schnelle Umsetzung von Kundenwünschen sowie die gesellschaftliche Verantwortung umfasst. Dies korrespondiert in Teilen mit den Ergebnissen von Winkelmann (2004); ihm zu Folge gelten Produktinnovationen, der organisatorische Aufbau des Unternehmens, dessen Produktionstechnologie, die Qualifikation der Mitarbeiter sowie Werbung als die fünf zentralen Erfolgsfaktoren in deutschen Molkereien. Gegenüber den Elementen allgemeiner Exzellenz-Modelle (siehe Kapitel 3) sind für Molkereien a) Rohstoffe für Molkereiprodukte, b) Lieferantenbeziehungen sowie c) die gesellschaftliche Verantwortungsübernahme weitere herauszustellende Bereiche. Die Einhaltung strenger Hygienerichtlinien ist dabei kein eigener Faktor von Exzellenz, sondern gilt als Selbstverständlichkeit.

Nach Dalluege et al. (2012) zeigt sich die Exzellenz von Organisationen in dauerhaft herausragenden Leistungen, die außerdem die Erwartungen aller Stakeholder erfüllen oder übertreffen. Produktattribute wie nachhaltig, regional 
oder biologisch fördern aus Sicht der Molkereivertretung in diesem Zusammenhang die Vermittlung von „emotionaler Qualität" von Milch und Milcherzeugnissen gegenüber den Konsumenten. Die befragten Experten der Molkereibranche berichten im Einzelnen von Wiesenmilch, Weidemilch, laktosefreier Milch oder weiteren Clean-Labeln; natürliche Produkte, schonende Verfahren, umweltfreundliche Verpackungen zählen als weitere kundenorientierte Themen exzellenter Molkereien. Mit Blick auf die Trendforschung oder in Fachmagazine stehen künstliche Milch aus dem Labor, vegetarisch-vegane Milchalternativen, koschere Produktionsverfahren oder Innovationen zur Lebensmittelverschwendung ebenfalls auf der Agenda der Branche, die im Interview - möglicherweise aus Gründen des Betriebsgeheimnisses - als weitere Ideen unbenannt blieben. Mit steigenden Produktanforderungen der Konsumenten (siehe Kapitel 3) sowie zunehmenden Möglichkeiten der digitalen Informationsbeschaffung wird es für exzellente Molkereien unumgänglich, sich durch hervorragende Leistungen und eine klare Positionierung am konkurrierenden Markt zu differenzieren oder Nischen zu belegen. Die Herausforderung hierin besteht a) in der permanenten Suche nach Neuem, b) in der Identifizierung von Kundenwünschen, die als weitere Attribute verlangt werden und Mehrwert versprechen, sowie c) deren kohärenten Verknüpfung mit bereits Bestehenden. Flexibilität, Innovationsfreudigkeit und der Wille zur kontinuierlichen Verbesserung sind dabei wesentliche Erfolgsfaktoren am Weg in eine exzellente Zukunft von Molkereien.

Bezüglich der zweiten Forschungsfrage, welche Stellenwerte Selbstberichte und Drittberichte, wie Prämierungen und Zertifizierungen, in der Kommunikation über Exzellenz von Molkereien einnehmen, zeigt sich, dass diesen unterschiedliche Bedeutung zuerkannt wird und sie dementsprechend in der Kommunikation der Molkereien eingesetzt werden. Grundsätzlich stimmen alle Experten überein, dass Exzellenz und in diesem Sinne produkt- und unternehmensspezifische Besonderheiten kommunikativ hervorgehoben werden müssen, um sich gegenüber dem Mitwettbewerb abzuheben und für Abnehmer sichtbar zu positionieren. Über das Ausmaß und die Wahl der Instrumente gibt es unterschiedliche Ansichten. Eine zentrale Rolle spielen hierbei verschiedene Unternehmensgrößen, je kleiner ein Unternehmen, desto weniger wird über Nachhaltigkeit, Prämierungen und Zertifizierungen kommuniziert. Gebhardt et al. (2019) stellen diese Kausalität bereits in Deutschland im Rahmen eines Branchenvergleiches von Molkereien und der Fleischverarbeitung fest.
Im Einzelnen sind es zuvorderst Selbstberichte, insbesondere mittels Internetpräsenzen, die in der Markt- und Unternehmenskommunikation der Ernährungswirtschaft eingesetzt werden (siehe Kapitel 4). Das Thema „Nachhaltigkeit", das in dieser Studie beispielhaft ausgewählt wurde, spielt aus Sicht der befragten Experten eine wesentliche Rolle in der Exzellenz von Molkereien. Dies korrespondiert mit der beobachteten Unternehmenskommunikation auf der Website: Über 68 Prozent der Molkereien in Österreich bzw. 50 Prozent in Deutschland berichten auf ihrer Internetseite über Nachhaltigkeit. Wie Studien für die deutsche Ernährungswirtschaft festhalten (Friedrich et al., 2013; ZNU, 2013), stellen Unternehmen der Molkereibranche hier teilweise nur oberflächliche Informationen oder zu nur einer Säule der Nachhaltigkeit zur Verfügung. Da sich hinter dem Begriff der Nachhaltigkeit nicht zwingend tatsächliches Engagement in Bezug auf alle drei Säulen der Nachhaltigkeit verbirgt, können vor allem in diesem Bereich entsprechende Prämierungen oder Zertifizierungen eine, wenn auch nur schwache Orientierungshilfe für die Konsumenten und Konsumentinnen bieten (Gebhardt, 2016) - auch da eine systematische Nachhaltigkeitskommunikation, beispielsweise in Form von Nachhaltigkeitsberichten, von weniger als einem Zehntel aller Molkereien in beiden Ländern veröffentlicht wird.

Branchen- oder themenspezifische Prämierungen bieten den Molkereien eine Möglichkeit, sich mit den Mitbewerbern in künstlichen Wettbewerben zu messen und von dritter Seite beurteilt, als „Bester“ zu präsentieren. Prämierungen gelten als aufmerksamkeitsfördernd, teils auch als kostengünstig. Auf ihren Websites berichten 54 Prozent der österreichischen und 42 Prozent der deutschen Molkereien über gewonnene Prämierungen, vor allem über Produktprämierungen. Produktprämierungen heben oftmals das den Verbrauchern wichtigste Attribut im Lebensmitteleinkauf, den Geschmack (MRI, 2008), hervor. Prämierungen haben eine herausragenden Stellenwert für österreichische Konsumenten: Nach Gruber et al. (2015) sind Awards die am glaubwürdigsten bewerten Instrumente der CSRKommunikation; für deutsche Konsumenten ordnet sich die Glaubwürdigkeit von Prämierungen in einem mittleren Bereich ein (Gebhardt, 2018). Eine zu hohe Zahl an Prämierungen - gleiches gilt auch für Zertifizierungen - führt auf der anderen Seite tendenziell zum Bedeutungsverlust und zur Überforderung der Konsumenten angesichts der "Siegelflut". Teilweise fehlt auch Molkereien das Wissen über geeignete und glaubwürdige Wettbewerbe (Gebhardt, 2018) oder Zertifizierungsmöglichkeiten. Daher gilt deren 
Transparenz und eine zielgruppenadäquate Wissensvermittlung gegenüber allen Stakeholdern sowohl bei Prämierungen als auch bei Zertifizierungen als essenziell.

Zertifizierungen gelten nur eingeschränkt als Zeichen für Exzellenz, Zertifikate stellen aus Sicht der Experten in vielen Fällen einen Standard dar (mit Ausnahme von Bio, Heumilch oder Gentechnik-frei) oder werden vom LEH (IFS, BRC) sowie den Kunden vorausgesetzt. Sie sind für die Molkereibranche dennoch sehr wichtig und bilden die Grundlage für exzellente Unternehmen. Demgegenüber sind Zertifizierungen für biologische Produkte in der Molkereibranche zunehmend verbreitet (BMLFUW, 2017; MIV, 2017), vor allem in kleinen Unternehmen, und bedeuten aus Expertensicht dort Exzellenz per se. Darüber hinaus werden sie mit ökologischen Aspekten der Nachhaltigkeit in Verbindung gebracht. Zertifikate für sozialökologische Managementsysteme (ISO 14001, EMAS) hingegen gelten als zeit- und kostenintensiv. Diese sind in beiden Ländern nur marginal verbreitet, besonders selten auf den Internetseiten kleiner Molkereien.

Herausfordernd für die vielen kleinen Unternehmen beider Länder bleibt, dass die Ausrichtung auf Nachhaltigkeit als bisher empfohlene Differenzierungsstrategie nun von den Großen ihrer Branche besetzt wird und diese dafür zunehmend - wie bereits für die Qualität ihrer Produkte - prämiert werden (Gebhardt et al., 2019). Erschwerend kommt hinzu, dass Exzellenz eine veränderliche Größe ist, die vom permanenten Verbesserungsprozess in den Unternehmen, dem Nachziehen der Mitwettbewerber und den Ansprüchen der Gesellschaft im Wandel der Zeit aufgehoben werden kann. Wenn jedoch alle Unternehmen versuchen, sich in die gleiche Richtung zu differenzieren, ist auf lange Sicht kein Abheben von der Konkurrenz möglich. Die dritte Frage, worin sich die Sichtweisen und Darstellung von Exzellenz in der österreichischen und der deutschen Molkereibranche unterscheiden, pointiert zuletzt den Ländervergleich. Zur Erinnerung: Exzellenz ist relativ und bedarf des Vergleichs, um sichtbar zu werden. Um sich als einzigartig abzuheben, sind die deutlichen Unterschiede wichtig, weniger die Gemeinsamkeiten (siehe Kapitel 3). Dies betrifft sowohl die einzelbetriebliche Ebene als auch die übergeordnete Ebene, den Gesamtmarkt für Molkereiprodukte im jeweiligen Land. Die Unterschiede bei Letzterem sind für die herausragende internationale Positionierung der jeweiligen Molkereibranche wichtig und basieren auf der Frage, was den besonderen Wert von Molkereiprodukten des jeweiligen Landes ausmacht.
Die befragten Experten beider Länder sind sich im Verständnis von Exzellenz in der Molkereibranche und deren Darstellung grundlegend einig. Die Unterschiede in Österreich und in Deutschland scheinen auf den ersten Blick marginal (siehe Kapitelteil 5.1.). Die Verbundenheit mit traditionellen Herstellungsverfahren, die überwiegend genossenschaftliche Struktur der Molkereien sowie die Besonderheiten der kleinteiligen Milchproduktion in benachteiligten Gebieten oder Bergregionen (siehe Kapitel 3) kennzeichnen die Situation der österreichische Molkereien, die in den exklusiv herausgestellten Kernbereichen von Exzellenz - die Herkunft des Futters (u. a. Heumilch) sowie faire Partnerschaften - zum Ausdruck kommen. Darauf fußt die kommunizierte „Authentizität“ der österreichischen Molkereiprodukte. Gleiches kann für die deutlich größere Molkereibranche in Deutschland mit rund zehnmal mehr Molkereien, einem höheren Anteil von großen Unternehmen und durchschnittlich höheren Tierbeständen (siehe Kapitel 3) nicht festgestellt werden, wenn man den Aussagen folgt, die alleine deutsche Experten anführen. Im Kernbereich von Exzellenz finden sich demnach Natur und Natürlichkeit, artgerechte Tierhaltung sowie Wachstumsstrategien. Diese als einzigartig verbleibenden Bereiche sind indes kaum belegbar oder gelten nicht für die gesamte deutsche Molkereibranche. Ein einheitliches Bild, was den einzigartigen Wert von Molkereiprodukten aus Deutschland ausmacht, lässt sich nicht ermitteln. Die Unterschiede liegen damit a) in der unterschiedlichen Struktur der betrachteten Länder mit deutlich mehr kleinen Molkereien in Österreich und einer größeren Heterogenität in Deutschland sowie b) einer für Deutschland länger beobachteten Low-Profile-Strategie (Wienert, 2007; ZNU, 2013; Rottwilm und Theuvsen, 2016).

Das Beispiel von zertifiziert gentechnikfreier Milch in Österreich verdeutlicht, dass Exzellenz über die Ausweitung als Standard auf alle Molkereien, im inländischen Markt nivelliert wird. Um sich als Einzelunternehmen im Inland (weiterhin) abheben zu können, sind zusätzliche Innovationen und Differenzierungen zwingend notwendig. In diesem Sinne kann ein inländischer Standard als Innovationstreiber verstanden werden. Eine solche inländische Standarderhöhung für alle Milcherzeugnisse bedeutet auch, dass sich Molkereien auf internationalen Märkten gegenüber der dortigen Konkurrenz - mit staatlicher Unterstützung als exzellent und einzigartig sichtbar abheben können. Es bleibt dennoch nur eine begrenzte Zeit, sich mit exzellenten Produkten oder Unternehmensaktivitäten 
zu positionieren bis der Mitwettbewerb nachzieht. Die Innovationsfähigkeit ist daher nicht nur eine zentrale, sondern eine dauerhafte Herausforderung, auch in der Kommunikation.

\subsection{Limitationen und weiterer Forschungsbedarf}

Die herangezogenen Studien zeigen mittels Experteninterviews die individuelle Sicht der ausgewählten Molkereivertretung auf Exzellenz und die Website-Analyse die externe Kommunikation darüber. Neben einer Quantifizierung der identifizierten Exzellenzfaktoren vonseiten der Molkereibranche und einem repräsentativen Ansatz, müsste für eine ganzheitliche Bewertung von Exzellenz auch geklärt werden, was Konsumenten unter Exzellenz verstehen und wie sie hierbei Molkereien und deren Produkte einordnen. Vertiefende Analysen zur Exzellenzkommunikation, auch weiterer Instrumente, sowie eine europäische Erweiterung der Untersuchungen versprechen außerdem eine differenzierte Basis für Handlungsempfehlungen der Molkereibranche in Österreich und in Deutschland.

\section{Literaturverzeichnis}

Albersmeier, F. und A. Spiller (2009): Das Ansehen der Fleischwirtschaft. Zur Bedeutung einer stufenübergreifenden Perspektive. In: Böhm, J., Albersmeier, F. und A. Spiller (Hrsg.): Die Ernährungswirtschaft im Scheinwerferlicht der Öffentlichkeit. Josef Eul, Lohmar, S. 213-250.

AMA - Agrarmarkt Austria (2019): RollAMA - Marktentwicklung Milch und Milchprodukte, 1. Quartal 2019. https://www.amainfo.at. Abgerufen am 24. Juli 2019.

ARGE Gentechnik-frei (2015): Erfolgsgeschichte: Gentechnik-freie Milch aus Österreich. http://www.gentechnikfrei.at/erfolgsgeschichte-gentechnik-freie-milchaus-oesterreich. Abgerufen am 28. Jänner 2018.

ARGE Heumilch (o. J.): Milch mit Tradition! http://www. heumilch.com. Abgerufen am 28. Mai 2019.

Axjonow, A., Ernstberger, J. und C. Pott (2016): Auswirkungen der CSR-Berichterstattung auf die Unternehmensreputation. uwf - UmweltWirtschaftsForum 24, 215-221.

Benes, G. und P. Groh (2012): Grundlagen des Qualitätsmanagements. 1. Aufl., Carl Hanser, München.

BMEL - Bundesministerium für Ernährung und Landwirtschaft (2017): Milchbericht 2017. https://www.
bmel.de/SharedDocs/Downloads/Broschueren/Milchbericht2017.pdf?_blob=publicationFile. Abgerufen am 4. Juli 2018.

BMELV - Bundesministerium für Ernährung, Landwirtschaft und Verbraucherschutz (2008): Strukturbericht Molkereiwirtschaft 2006. http://www.bmelv-statistik. de. Abgerufen am 14. April 2016.

BMi (2014): Milcherzeuger profitieren von Genossenschaften. MilchPartner 3, 4-5.

Herzog, U. (2015): Wo stehen wir in Österreich und der EU - Brennpunkte im Tierschutz. https://www.lko.at/ media.php? filename=download\%3D\%2F2 015 . $11.10 \% 2 \mathrm{~F} 1447153382071299 \cdot \mathrm{pdf} \& \mathrm{rn}=\operatorname{Pr} \% \mathrm{E} 4$ sentation\%20Dr.\%20Herzog_Brennpunkt\%20Tierschutz.pdf. Abgerufen am 05. Oktober 2016.

BMLFUW - Bundesministerium für Land- und Forstwirtschaft, Umwelt und Wasserwirtschaft (2016): Grüner Bericht 2016. Selbstverlag, Wien.

BMLFUW - Bundesministerium für Land- und Forstwirtschaft, Umwelt und Wasserwirtschaft (2017): Grüner Bericht 2017. Selbstverlag, Wien.

Brugger, F. (2010): Nachhaltigkeit in der Unternehmenskommunikation: Bedeutung, Charakteristika und Herausforderungen. Gabler, Wiesbaden.

Brunner, F. und K. Wagner (2011): Taschenbuch Qualitätsmanagement: Leitfaden für Studium und Praxis. 5., überarb. Aufl., Hanser, München - Wien.

Collins, J. (2001): Good to great. Why some companies make the leap... and others don't. Harper-Collins: New York.

Cronin, B. und M. Gudim (1987): Excellence under the microscope. Aslib Proceedings 39, 17-31.

Dahlgaard-Park, S. und Dahlgaard, J. (2006): In Search of Excellence - Past, Present and Future. In: Schnauber, H. (Hrsg.): Kreativ und konsequent. München, Carl Hanser, S. 57-84.

Dalluege, C., Franz, H., Pfeffer, W und H. Schneider (2012): Exzellenz durch nachhaltige Unternehmensstrategien. Dr. Curt Haefner, Heidelberg.

DRV - Deutscher Raiffeisenverband e.V. (2017): Milchgenossenschaften. http://www.raiffeisen.de/uebersichtder-genossenschaftssparten/milch/. Abgerufen am 26. März 2018.

Europäische Kommission (2003): Empfehlung der Kommission vom 6. Mai 2003 betreffend die Definition der Kleinstunternehmen sowie der kleinen und mittleren Unternehmen. Selbstverlag, Brüssel. 
Europäische Kommission (2011): Eine neue EU-Strategie (2011-14) für die soziale Verantwortung der Unternehmen (CSR). Selbstverlag, Brüssel.

Flint, L., Kuhnert, H., Laggner, B., Lassen, B., Nieberg, H. und R. Strohm (2016): Prozess nachhaltige Milcherzeugung - Entwicklung eines Nachhaltigkeitsmoduls zur Erfassung und Bewertung von Nachhaltigkeitskriterien auf milchviehhaltenden Betrieben. Thünen Working Paper 54, Braunschweig.

Forsa (2017): Meinungen und Einstellungen der Bürger zur Milchwirtschaft in Deutschland 2017. http:// dialog-milch.de/wp-content/uploads/2017/05/34619_ Q7381_Einstellungen-zur-Milchwirtschaft_2017.pdf. Abgerufen am 26. März 2018.

Frey, B.S. und J. Gallus (2014): The power of Awards. The Economists's Voice 11, 1-5.

Friedrich, C. (2010): Milchverarbeitung und -vermarktung in Deutschland. https://www.econstor.eu/bitstream/10419/41464/1/627385095.pdf. Abgerufen am 26. März 2018.

Friedrich, N., Wellner, M. und L. Theuvsen (2013): Nutzung des Internets für die Nachhaltigkeitsberichterstattung in der Ernährungsbranche. In: Clasen, M., Kersebaum, K. C., Meyer-Aurich, A. und B. Theuvsen (Hrsg.): Massendatenmanagement in der Agrar- und Ernährungswirtschaft. Gesellschaft für Informatik, Bonn, S. 83-86.

Gebhardt, B. (2012): Akzeptanz und Erfolg kleinräumiger Systeme der Lebensmittelversorgung im urbanen Umfeld am Beispiel Stuttgart - Empirische Untersuchungen von Verbrauchern und Unternehmen. Hohenheimer Agrarökonomischer Arbeitsbericht Nr. 22. Universität Hohenheim.

Gebhardt, B. (2016): Ausgezeichnet! - Nachhaltigkeitspreise für Unternehmen der deutschen Ernährungswirtschaft. Dr. Kovač, Hamburg.

Gebhardt, B. (2018): Nachhaltigkeitsawards - Ein politisches Instrument der individuellen Verhaltensbeeinflussung? Vierteljahreshefte zur Wirtschaftsforschung, 87(1), 133-153.

Gebhardt, B., Ding, J. und T. Strohäker (2019): Besser, schneller, nachhaltiger: Exzellenzkommunikation in der deutschen Milch- und Fleischverarbeitung im Branchenvergleich. Austrian Journal of Agricultural Economics and Rural Studies, 28(12), DOI 10.15203/OEGA_28.12.

GRI - Global Reporting Initiative (2015): G4 Leitlinien zur Nachhaltigkeitsberichterstattung. Selbstverlag, Amsterdam.
Gruber, V., Kaliauer, M. und B. Schlegelmilch (2015): Improving the Effectivenness and Credibility of Corporate Scocial-Responsibility Messaging. Journal of Adverting Research, 15, 1-13.

Hahn, R. und R. Lülfs (2014) Legitimizing negative aspects in GRI-oriented sustainability reporting, Journal of business ethics, 123(3), 401-420.

Hansen, L. und B. Weisbrod (1972): Towards a General Theory of Awards, or, Do Economists Need a Hall of Fame? The Journal of Political Economy 80, 22-431.

Huck-Sandhu, S. (2011): Corporate Social Responsibility und internationale Public Relations. In: Raupp, J., Jarolimek, S. und F. Schultz (Hrsg.): Handbuch CSR -Kommunikationswissenschaftliche Grundlagen, disziplinäre Zugänge und methodische Herausforderungen. VS Verlag für Sozialwissenschaften, Wiesbaden, S. 205-228.

Hutton, B. und D. Mayer (2010): Corporate Social Responsibility and Corporate Excellence. In: O’Toole, J. und D. Mayer (Hrsg.): Good Business - Exercising effective and ethical leadership. Routledge, New York, 94-106.

Imhof, K. (2009): Exzellenz im Krieg der Märkte. In: Surdez, M. und C. Suter (Hrsg.): Die Besten - Auf dem Gipfel der Exzellenz. Seismo, Zürich, S. 4-8.

Johnson, D. (2006): Corporate Excellence, Ethics and the role of it. Business \& Society Review 111, 457-470.

Keunecke, K. (2017): Gentechnikfreie Milch wächst dynamisch. https://www.ami-informiert.de. Abgerufen am 24. Juli 2019.

Kirchhoff, K. (2006): CSR als strategische Herausforderung. In: Gazdar, K., Habisch, A., Kirchhoff, K. R. und S. Vaseghi (Hrsg.): Erfolgsfaktor Verantwortung. Springer, Berlin - Heidelberg.

Kurz, R. und W. Wild (2015): Nachhaltigkeit und Unternehmen. uwf - UmweltWirtschaftsForum, 23, 323328.

LEL - Landesanstalt für Entwicklung der Landwirtschaft und der Ländlichen Räume (2016): Agrarmärkte 2016. Selbstverlag, Schwäbisch Gmünd.

Lendle, M. (2012): Nachhaltigkeit in der Ernährungsbranche: Strategien sicher planen und umsetzen. 1. Aufl., Behr's, Hamburg.

Luhmann, H., C. Schaper, L. C., Theuvsen, L. und I. Weiland (2017): Was bedingt die Bereitschaft deutscher Milcherzeuger zur Teilnahme an einem Nachhaltigkeitsstandard? Ergebnisse einer empirischen Untersuchung. Schriften der Gesellschaft für Wirtschafts- und Sozialwissenschaften des Landbaues e. V., Band 52, 281-293. 
Mast, C. (2013): Unternehmenskommunikation. Ein Leitfaden. UVK: Konstanz/München.

Mayring, P. (2015): Qualitative Inhaltsanalyse: Grundlagen und Techniken. 12., überarbeitete Aufl., Beltz, Weinheim.

Meffert, H., Burmann, C. und M. Kirchgeorg (2018): Marketing: Grundlagen marktorientierter Unternehmensführung Konzepte - Instrumente - Praxisbeispiele. 13. Aufl., Springer Gabler, Wiesbaden.

Meyer-Höfer, M. und A. Spiller (2016): Strategien und Erfolgskriterien für Zertifikatsysteme am Beispiel der Agrar- und Ernährungswirtschaft. In: Friedel, R. und E. A. Spindler (Hrsg.): Zertifikate als Erfolgsfaktor. Springer Gabler, Wiesbaden, S. 75-88.

MIV - Milchindustrie-Verband (2016): TOP 10 Molkereien in Deutschland 2016. http://milchindustrie. de/uploads/tx_news/TOP_Molkereien_DE_Homepage_01.pdf. Abgerufen am 28. Jänner 2018.

MIV - Milchindustrie-Verband (2017): Milch und mehr: Die deutsche Milchwirtschaft auf einen Blick. Selbstverlag, Berlin.

Moog, K. und B. Gebhardt (2018): Regionalität im Verständnis von Verbrauchern und Zeichenträgern: ein Ländervergleich für Schweinefleisch. Bericht über Landwirtschaft 96, 1-34.

MRI - Max-Rubner-Institut (2008): Nationale Verzehrsstudie II. Ergebnisbericht, Karlsruhe.

Nieberg, H. und R. Schleenbecker (2014): Nachhaltige Milchproduktion - Wer ist wo und wie national und international unterwegs? Vortrag im Rahmen der DBU Sommerakademie vom 01.07.2014 in St. Marienthal.

Pöchtrager, S. (2011): Qualitätsmanagement in der Agrarund Ernährungswirtschaft: Institutionen, Strukturen und entscheidungsrelevante Faktoren. Springer, Wien.

Porter, M. (1985): Competitive advantage. Creating and sustaining superior performance. 14. Auflage. Free Press, New York.

Pufé, I. (2014): Nachhaltigkeit. 2. überarb. u. erw. Aufl., UTB, Konstanz - Stuttgart.

RNE - Rat für nachhaltige Entwicklung (2014): EU beschließt nicht-finanzielle Berichts-pflicht für große Unternehmen. http://www.nachhaltigkeitsrat.de/newsnachhaltigkeit/2014/2014-09-10/eu-beschliesstnicht-finanzielle-berichtspflicht-fuer-grosseunternehmen/?size=irbaocsc. Abgerufen am 25. November 2014.

Rocha-Lona, L., Garza-Reyes, J., Lim, M. K. und V. Kumar (2015): Corporate Sustainability and Business
Excellence. In: IEOM (Hrsg.): International Conference on Industrial Engineering and Operations Management. Piscataway, NJ, S. 1-7.

Rottwild, I. und L. Theuvsen (2016): Nachhaltigkeitskommunikation in der Ernährungswirtschaft. In: Willers, C. (Hrsg.): CSR und Lebensmittelwirtschaft. Nachhaltiges Wirtschaften entlang der Food Value Chain. Berlin/ Heidelberg, Springer, S. 119-137.

Sass, J. (2014): Analyse und Optimierung von Kommunikationsstrukturen: Audits und Exzellenzmodelle. In: Zerfaß, A. und M. Piwinger (Hrsg.): Handbuch Unternehmenskommunikation. Springer Fachmedien, Wiesbaden, S. 1063-1076.

Schmid, E., Larcher, M., Schönhart, M. und C. Stiglbauer (2011): Ende der Milchquote - Perspektiven und Ziele österreichischer Molkereien und MilchproduzentInnen. Forschungsbericht im Auftrag des BMLFUW. https:// wpr.boku.ac.at/wpr_dp/DP-52-2011.pdf. Abgerufen am 28. Jänner 2018.

Statistisches Bundesamt (2008): Klassifikation der Wirtschaftszweige (WZ 2008). URL: https://www.destatis. del. Abgerufen am 11. April 2016.

Statistisches Bundesamt (2018): Milchkuhbestand in Deutschland nach Bundesländern im Jahr 2017. http:// de.statista.com. Abgerufen am 4. Juli 2018.

Steffens, N. (2013): Supply Chain Management im Agribusiness: Herausforderungen für Molkereigenossenschaften. 1. Aufl., Cuvillier, Göttingen.

Töpfer, A. (2006): Audit von Business Excellence. Ganzheitliche strategische und operative Steuerung in der marktorientierten Unternehmensführung. In: Reinecke, S. (Hrsg.): Handbuch Marketingcontrolling. Springer Fachmedien, Wiesbaden, 117-154.

Tribl, C. und K. Salhofer (2013): Marktmacht und räumlicher Wettbewerb entlang der Wertschöpfungskette von Milch: Abschlussbericht zu Projekt AWI/158/06 „Industrieökonomische Analysen der Sektoren Landwirtschaft und Lebensmittelverarbeitung“. Bundesanstalt für Agrarwirtschaft, Wien.

Umweltpakt Bayern (2012): EMAS: Das Umweltmanagement der EU in der Praxis. https://www.ihk-muenchen.de/ ihk/documents/Gr\%C3\%BCndung/Umweltmanagement/ EMAS-Leitfaden.pdf. Abgerufen am 22. Februar 2017.

Veer, B. und P. Teitscheid (2010a): Wie nachhaltig sind Molkereien? - Teil 1. Deutsche Molkerei Zeitung 2, 22-24.

Veer, B. und P. Teitscheid (2010b): Wie nachhaltig sind Molkereien? - Teil 2. Deutsche Molkerei Zeitung 22, 20-22. 
Voeth, M. und U. Herbst (2013): Marketing-Management. Grundlagen, Konzeption und Umsetzung. Schäffer-Poeschel, Stuttgart.

VÖM - Vereinigung Österreichischer Milchverarbeiter (2016): GTS-Auszeichnung für Heumilch unterstreicht Qualität der heimischen Milchprodukte. http://www.voem.or.at/index.php?id=31\&no_ cache $=1 \&$ tx_news_pi $1 \% 5 \mathrm{Bnews} \% 5 \mathrm{D}=85 \& \mathrm{tx} \_$news_ pi $1 \% 5 \mathrm{~B}$ controller\%5D=News\&tx_news_pi $1 \% 5 \mathrm{Bactio}$ $\mathrm{n} \% 5 \mathrm{D}=$ detail \&cHash=971 e66d9ce7ea4f9e05c6d76e8 c5fdaf. Abgerufen am 28. Jänner 2018.

von Alvensleben, R. (2000): Zur Bedeutung von Emotionen bei der Bildung von Präferenzen für regionale Produkte. Agrarwirtschaft 49, 399-402.

Weindlmaier, H. (2004): Qualitätsmanagementsysteme in der Ernährungswirtschaft: Beweggründe, Entwicklungen und Perspektiven. Jahrbuch der Österreichischen Gesellschaft für Agrarökonomie 14, 7-26.

Wienert, M. (2007): Integrierte Kommunikation in Milch verarbeitenden Unternehmen. Dissertation. Fakultät für Wirtschaftswissenschaften, Universität München.
Winkelmann, T. (2004): Erfolgsfaktoren in der Molkereiwirtschaft. Dissertation. Fakultät für Wirtschaftswissenschaften, Universität München.

Woywode, M. (2004): Wege aus der Erfolglosigkeit der Erfolgsfaktorenforschung. In: KfW Bankengruppe (Hrsg.): Was erfolgreiche Unternehmen ausmacht. Erkenntnisse aus Wissenschaft und Praxis. Physica, Heidelberg, S. 15-48.

Wunder, T. und J. Bausch (2014): Ökologische Nachhaltigkeit in der Ernährungsindustrie. In: Ökologisches Wirtschaften 29, S. 44-50.

Wunder, T. und J. Bausch (2015): Auf dem Weg zur operativen Exzellenz. Erfolgsfaktoren und Handlungsempfehlungen. Controller Magazin 06/2015, 53-61.

Zander, K. (2016): „Ethischer Konsum“ Anforderungen an Umwelt- und Sozialzertifikate bei Lebensmitteln. In: Friedel, R. und E. A. Spindler (Hrsg.): Zertifikate als Erfolgsfaktor. Springer Gabler, Wiesbaden, S. 277-285.

ZNU - Zentrum für nachhaltige Unternehmensführung (2013): Nachhaltigkeit und Nachhaltigkeitskommunikation - Wo steht die Lebensmittelwirtschaft? http://mehrwert-nachhaltigkeit.de. Abgerufen am 7. August 2014. 